This item was submitted to Loughborough's Research Repository by the author.

Items in Figshare are protected by copyright, with all rights reserved, unless otherwise indicated.

\title{
Memristors with diffusive dynamics as synaptic emulators for neuromorphic computing
}

PLEASE CITE THE PUBLISHED VERSION

http://dx.doi.org/10.1038/nmat4756

\section{PUBLISHER}

(C) Nature Publishing Group

\section{VERSION}

AM (Accepted Manuscript)

\section{PUBLISHER STATEMENT}

This work is made available according to the conditions of the Creative Commons Attribution-NonCommercialNoDerivatives 4.0 International (CC BY-NC-ND 4.0) licence. Full details of this licence are available at: https://creativecommons.org/licenses/by-nc-nd/4.0/

\section{LICENCE}

CC BY-NC-ND 4.0

\section{REPOSITORY RECORD}

Wang, Zhongrui, Saumil Joshi, Sergey Saveliev, Hao Jiang, Rivu Midya, Peng Lin, Miao Hu, et al.. 2016. "Memristors with Diffusive Dynamics as Synaptic Emulators for Neuromorphic Computing". Loughborough University. https://hdl.handle.net/2134/23851. 
Memristors with diffusive dynamics as synaptic emulators for neuromorphic computing

Zhongrui Wang ${ }^{1 \dagger}$, Saumil Joshi ${ }^{1 \dagger}$, Sergey E. Savel'ev ${ }^{2}$, Hao Jiang ${ }^{1}$, Rivu Midya ${ }^{1}$, Peng Lin $^{1}$, Miao $\mathrm{Hu}^{3}$, Ning $\mathrm{Ge}^{3}$, John Paul Strachan ${ }^{3}$, Zhiyong $\mathrm{Li}^{3}$, Qing $\mathrm{Wu}^{4}$, Mark Barnell ${ }^{4}$, Geng-Lin $\mathrm{Li}^{5}$, Huolin L. Xin ${ }^{6}$, R. Stanley Williams ${ }^{3}$, Qiangfei Xia ${ }^{1}$, and J. Joshua Yang ${ }^{1 *}$

${ }^{1}$ Department of Electrical and Computer Engineering, University of Massachusetts, Amherst, MA 01003, USA

${ }^{2}$ Department of Physics, Loughborough University, Loughborough LE11 3TU, UK

${ }^{3}$ Hewlett Packard Labs, Palo Alto, California 94304, USA

${ }^{4}$ Air Force Research Lab, Information Directorate, Rome, New York 13441, USA.

${ }^{5}$ Department of Biology, University of Massachusetts, Amherst, MA 01003, USA

${ }^{6}$ Center for Functional Nanomaterials, Brookhaven National Laboratory, Upton, New York 11973, USA

${ }^{\dagger}$ These authors contributed equally to this work.

*e-mail: jjyang@umass.edu

\section{Abstract}

The accumulation and extrusion of $\mathrm{Ca}^{2+}$ in the pre- and postsynaptic compartments play a critical role in initiating plastic changes in biological synapses. To emulate this fundamental process in electronic devices, we developed diffusive Ag-in-oxide memristors with a temporal response during and after stimulation similar to that of the synaptic $\mathrm{Ca}^{2+}$ dynamics. In situ high-resolution transmission electron microscopy and nanoparticle dynamics simulations both demonstrate that Ag atoms disperse under electrical bias and regroup spontaneously under zero bias because of interfacial energy 
minimization, closely resembling synaptic influx and extrusion of $\mathrm{Ca}^{2+}$, respectively. The diffusive memristor and its dynamics enable a direct emulation of both short- and long-term plasticity of biological synapses and represent a major advancement in hardware implementation of neuromorphic functionalities.

CMOS circuits have been employed to mimic synaptic $\mathrm{Ca}^{2+}$ dynamics, but three-terminal devices bear limited resemblance to bio-counterparts at the mechanism level and require significant numbers and complex circuits to simulate synaptic behavior ${ }^{1-3}$. A substantial reduction in footprint, complexity and energy consumption can be achieved by building a two-terminal circuit element, such as a memristor directly incorporating $\mathrm{Ca}^{2+}$-like dynamics. Various types of memristors based on ionic drift (drift-type memristor) ${ }^{4-8}$ have recently been utilized for this purpose in neuromorphic architectures ${ }^{9-15}$. Although qualitative synaptic functionality has been demonstrated, the fast switching and non-volatility of drift memristors optimized for memory applications do not faithfully replicate the nature of plasticity. Similar issues also exist in MOS-based memristor emulators ${ }^{16-18}$, although they are capable of simulating a variety of synaptic functions including spike-timing-dependent plasticity (STDP). Recently, Lu's group adopted second-order drift memristors to approximate the $\mathrm{Ca}^{2+}$ dynamics of chemical synapses by utilizing thermal dissipation ${ }^{19}$ or mobility decay ${ }^{20}$, which successfully demonstrated STDP with non-overlapping spikes and other synaptic functions, representing a significant step towards bio-realistic synaptic devices. This approach features repeatability and simplicity, but the significant differences of the dynamical response from actual synapses limit the fidelity and variety of desired synaptic functions. A device with similar physical behavior as the biological $\mathrm{Ca}^{2+}$ dynamics would enable improved emulation of synaptic function and broad applications to neuromorphic computing. Here we report such an emulator, which is a memristor based on metal atom 
diffusion and spontaneous nanoparticle formation, as determined by in situ high-resolution transmission electron microscopy (HRTEM) and nanoparticle dynamics simulations. The dynamical properties of the diffusive memristors were confirmed to be functionally equivalent to $\mathrm{Ca}^{2+}$ in bio-synapses, and their operating characteristics were experimentally verified by demonstrating both short- and long-term plasticity, including mechanisms that have not been unambiguously demonstrated previously.

The diffusive memristor illustrated in Figure 1a consists of two Au electrodes sandwiching a switching layer of a dielectric film with embedded Ag nanoclusters (Methods). X-ray photoelectron spectroscopy (XPS) revealed that the Ag was metallic, which was further confirmed by HRTEM micrographs showing Ag nanocrystals in $\mathrm{SiO}_{\mathrm{x}} \mathrm{N}_{\mathrm{y}}$ :Ag (Supplementary Fig. S1-S2). These devices are similar to electrochemical metallization memory (ECM) ${ }^{21-26}$ cells in terms of utilizing mobile species of noble metals, but they differ substantially in terms of the structural symmetry and operating voltage polarities, metal concentration and profile, and transient switching behaviour. An applied voltage above an apparent threshold abruptly switched the device to a conductance state limited by an external compliance current (Fig. 1b). To demonstrate that the device spontaneously relaxed back to an insulating configuration upon removing the bias (without applying an opposite polarity voltage), repeatable I-V loops with only positive applied voltages were used in collecting the data in Fig. 1b. Symmetric hysteresis loops were observed with the opposite polarity bias (Supplementary Fig. S3), showing that the threshold switching is unipolar in nature and significantly different from non-volatile drift-type memristors, especially in the OFF-switching process. The microdevices represented by Fig. 1 a have an area of $10 \mu \mathrm{m} \times 10 \mu \mathrm{m}$ and nano-devices with an area of 100nm×100nm exhibited similar switching behaviours (Supplementary Fig. S4). The resistance ratio between the conducting and insulating states was 5-orders of magnitude in 
$\mathrm{SiO}_{\mathrm{x}} \mathrm{N}_{\mathrm{y}}$ :Ag and over 10 -orders in $\mathrm{HfO}_{\mathrm{x}}$ :Ag devices, the highest reported in threshold switching devices so $\operatorname{far}^{27-30}$. The volatile switching had sharp turn-on slopes of $\sim 10 \mathrm{mV} /$ decade in $\mathrm{MgO}_{\mathrm{x}}: \mathrm{Ag}$ and $\mathrm{SiO}_{\mathrm{x}} \mathrm{N}_{\mathrm{y}}$ :Ag, and an extraordinary $\sim 1 \mathrm{mV} /$ decade in $\mathrm{HfO}_{\mathrm{x}}$ :Ag, the sharpest demonstrated to date ${ }^{27-30}$. The high current capability and large resistance ratio enable diffusive memristors to be utilized as selectors for mitigating sneak current paths in crossbar arrays ${ }^{27-30}$. In Fig. 1c, each $500 \mathrm{~ns} / 1.5 \mathrm{~V}$ switching pulse was followed by a $500 \mathrm{~ns} / 0.2 \mathrm{~V}$ reading pulse to verify that the device had relaxed back to the insulating state under zero bias within $5 \mu$ s after switching to the high conductance state. Repeatable and symmetric switching was demonstrated using wider bipolar voltage pulses with over a million switching cycles in Fig. 1d.

To examine the switching mechanism, especially the spontaneous relaxation to the insulating state upon ceasing power, a planar $\mathrm{Au} / \mathrm{SiO}_{\mathrm{x}} \mathrm{N}_{\mathrm{y}}: \mathrm{Ag} / \mathrm{Au}$ device with a nano-junction was fabricated for in-situ HRTEM characterizations (Supplementary Fig. S5a). Reliable threshold switching was observed under a 100nA compliance in ambient conditions with a typical relaxation time constant of $\sim 11 \mathrm{~ms}$ (Supplementary Fig. S5b-c). In the time sequence of HRTEM images in Fig. 2 (Supplementary Videos), the gap between the Au electrodes first experienced a constant voltage (20V) with an 100nA compliance from 0s to 5s, after which the power was turned off. We observed a delay time of $\sim 2$ s during which the measured current was $<5 n \mathrm{~A}$ and $\mathrm{Ag}$ nanoparticles formed in the gap region (indicated by the orange and blue arrows at time 0.1s), followed by an abrupt current jump to the compliance level as nanoparticles grew further to bridge the gap between the electrodes (indicated by the red arrow at $2.5 \mathrm{~s})$. At $4.6 \mathrm{~s}$, the cluster reached a diameter of $\sim 4.2 \mathrm{~nm}$. 
Up to this point, the behaviour of $\mathrm{Ag}$ nanoparticles in $\mathrm{SiO}_{\mathrm{x}} \mathrm{N}_{\mathrm{y}}$ is similar to previous observations, which have been interpreted as electrochemical reactions at effectively bipolar electrodes $^{21-26}$. We next turned off the power at 5.0s to observe the spontaneous relaxation, which is critical for understanding the dynamics of these devices, but has not been previously reported to the best of our knowledge. The elongated cluster of nanoparticles that had likely formed the conductive bridge rapidly contracted from a length of $14.8 \mathrm{~nm}$ to a circular profile with a diameter of $7.6 \mathrm{~nm}$ by $5.7 \mathrm{~s}$, indicating Ostwald ripening ${ }^{31}$. These observations demonstrated that minimizing the interfacial energy between the Ag nanoparticles and the dielectric served as the driving force for the relaxation dynamics of these diffusive memristors. The material systems exhibiting a substantial relaxation were those with large wetting contact angles ${ }^{32}$, such as $\mathrm{MgO}_{\mathrm{x}}$ : $\mathrm{Ag}, \mathrm{SiO}_{\mathrm{x}} \mathrm{N}_{\mathrm{y}}$ :Ag, and $\mathrm{HfO}_{\mathrm{x}}$ : $\mathrm{Ag}$ in Fig. 1b, consistent with the reported psudoelasticity of silver nanoparticles ${ }^{33}$ and the hypothesis that interfacial energy facilitates filament rupture in volatile switching ${ }^{34-36}$.

The dynamical properties of diffusive memristors were further studied by applying voltage pulses and measuring resulting currents. Under an applied pulse, the device exhibited threshold switching to a low resistance state after an incubation period $\tau_{d}$, as shown in Fig. 3a. This $\tau_{d}$ is related to the growth and clustering of silver nanoparticles to eventually form conduction channels. Upon channel formation, the current jumped abruptly by several orders of magnitude, and then slowly increased further under bias as the channel thickened. As the voltage pulse ended, the device relaxed back to its original high resistance state over a characteristic time $\tau_{r}$. As shown in Fig. $3 \mathrm{~b}, \tau_{r}$ decreased as the ambient temperature increased, consistent with a diffusion activation energy of $0.27 \mathrm{eV}$ (inset of Fig. 3b), and the characteristic time was on the same order as the response of bio-synapses, i.e., tens of ms. In addition to the temperature, $\tau_{d}$ and $\tau_{r}$ were also functions of the voltage pulse parameters, 
operation history, Ag concentration, host lattice, device geometry, humidity, and other factors $^{37-40}$, which alone or combined could be used to tune the desired dynamics for neuromorphic systems (Supplementary Fig. S6).

To better understand the switching mechanism, we performed simulations using a generalized model similar to the one utilized for non-volatile switching and current noise in $\mathrm{TaO}_{\mathrm{x}}$ memristors ${ }^{41}$. This model links electrical, nano-mechanical and thermal degrees of freedom (Methods). The model results here did not include redox reactions, although they can be added in order to more closely resemble the electrochemical models proposed previously ${ }^{21-26}$.

In our simulations, two large clusters of metallic nanoparticles are located near each terminal of the device (Fig. 4a1). When a voltage pulse is applied (Fig. 4a), the local temperature increases due to Joule heating and the potential is tilted by electric forces acting on particles with induced charge, both of which cause larger clusters to break up. As the nanoparticles become more uniformly distributed in the gap, the resistance drops, the current and temperature increase, and a positive feedback results in the formation of a conductive channel (Fig. 4a2). As soon as the power is turned off, the temperature drops, and the nanoparticles start to coalesce (Fig. 4a3), i.e. particles slowly diffuse to their minimum energy positions near the device terminals. Eventually, most of the nanoparticles have merged into larger clusters to minimize interfacial energy, and the high resistance state is re-established along with the original particle distribution almost restored (compare Fig. 4a1 and Fig. 4a4), leading to the observed volatility in Fig. 2. The model predicts interesting conductance evolution similar to synaptic behavior when a train of pulses is applied (Fig. 4b). First, when the initial voltage pulse is applied, electric field-assisted diffusion pumps some of the Agparticles out of the 'left' cluster and they start to bridge terminals. However, a single short 
pulse cannot excite enough particles to form a complete conducting path between the two terminals (Fig 4b2). If a subsequent pulse arrives before particles are re-absorbed, i.e., if the time between pulses is shorter than the diffusion relaxation time, more particles are pushed into the gap between terminals resulting in a gradual increase in device conductance, similar to the paired-pulse facilitation (PPF) phenomenon in bio-synapses. The result is that when high frequency pulses are applied, the device conductance increases with the number of pulses (Fig. 4b) until a conducting bridge is formed (Fig. 4b1-b4). Second, as the electric field pumps more and more particles towards one of the device terminals, the number of particles at the other terminal decreases (Fig 4b4-4b5, where the distribution peak at the left terminal decreases as more and more pulses arrive). As a result, the number of particles in the gap decreases (Fig 4b5-4b6) and the device conductance starts to decay. This results in an inflection of the device conductance due to excessive stimulation, capturing another synaptic behaviour, i.e., PPF followed by PPD (paired pulse depression). Third, sequential high voltage pulses with a long enough interval (low frequency) may form a conducting bridge first, but before the next pulse arrives the bridge breaks and the particles are re-absorbed at the terminals. Due to the electric field, the Ag particles gradually deplete at one terminal and accumulate at the other. Consequently, the conductance of the device starts to decrease from the initial state without showing facilitation first (PPD). (Supplementary Fig. S7c).

The simulations for Ag in dielectric agreed well with the experimental HRTEM observations. In addition, significant similarities exist between the Ag dynamics and that of synaptic $\mathrm{Ca}^{2+}$, not only in the diffusion mechanism but also in their dynamical balance of concentration and regulating roles in their respective systems. $\mathrm{Ca}^{2+}$ dynamics is responsible for initiating both short- and long-term plasticity of synapses, forming the basis of memory and learning ${ }^{42-44}$. In chemical synapses, the dynamical balance of the $\mathrm{Ca}^{2+}$ concentration is shaped by both influx 
via voltage-sensitive calcium channels (VSCC) and N-methyl-D-aspartate receptors $(\mathrm{NMDAR})^{42,43}$, and extrusion via the plasma membrane $\mathrm{Ca}^{2+}$-ATPase (PMCA) and $\mathrm{Na}^{+} / \mathrm{Ca}^{2+}$ exchanger $(\mathrm{NCE})^{43,44}$ that restores $\left[\mathrm{Ca}^{2+}\right]_{\mathrm{i}}$ to the basal concentration. The $\mathrm{Ca}^{2+}$ dynamics naturally leads to short-term plasticity in which residual elevation of presynaptic $\left[\mathrm{Ca}^{2+}\right]_{\mathrm{i}}$ directly correlates to the enhancement of synaptic transmission ${ }^{43-45}$. The calcium inside the postsynaptic membrane also plays important regulating roles in long-term potentiation and depression where $\mathrm{Ca}^{2+}$ accumulation is necessary to regulate enzymes, i.e. $\mathrm{Ca}^{2+} /$ calmodulindependent protein kinase II (CaMKII), calcineurin, and protein phosphatase $1^{42,43,46,47}$, which in turn triggers rapid and persistent modification of synaptic strengths by changing the number and/or conductance of $\alpha$-amino-3-hydroxy-5-methyl-4-isoxazolepropionic acid receptors (AMPAR) ${ }^{43,46,47}$. The Ag diffusion into the gap region between Ag nanoclusters with field assistance (Fig. 2 and Fig. 4), resembles the influx processes of $\mathrm{Ca}^{2+}$. The clearance of bridging Ag nanoparticles from the gap region by Ostwald ripening when electrical stimulus is removed replicates the extrusion processes of $\mathrm{Ca}^{2+}$. Thus, the Ag dynamics of the diffusive memristor is a functional emulation of bio-synapses (Fig. 5a). An expected characteristic of diffusive memristors is short-term plasticity, where application of paired pulses to synapses can induce an increase or decrease in postsynaptic responses, depending on the frequency of applied pulses. As shown in Fig. 5b, when the time interval between pulses ( $t_{\text {zero }}$ ) is short (high frequency), the device conductance increases (PPF) from its initial conductance (steady state of the diffusive memristor) as the number of pulses increases. In contrast, a long $t_{\text {zero }}$ (low frequency) leads to a reduced rate of increment or even a decrease in conductance (PPD) from the same initial conductance (Inset of Fig. 5b and Supplementary Fig. S7a-b) ${ }^{45,48}$. Moreover, it has also been shown in bio-synapses that prolonged or excessive stimulations with high-frequency (short $t_{\text {zero }}$ ) pulses will eventually lead to an inflection from facilitation to depression, an effect solely induced by an increased number of stimulation 
pulses at the same frequency ${ }^{45}$. This important feature of bio-synapses, which has not been clearly demonstrated previously on two terminal devices, was predicted in Fig. 4b and observed experimentally in Fig. 5c. The device in its steady state shows PPD upon low frequency $(196 \mathrm{~Hz})$ stimulation and experiences an increase in current (facilitation) once the stimulation frequency is raised $(5000 \mathrm{~Hz})$. The facilitation turns into depression (current decrease) with more pulses having identical frequency $(5000 \mathrm{~Hz})$ due to the gradual depletion of silver at one electrode and accumulation at the other. The depression continues with the low-frequency $(196 \mathrm{~Hz})$ pulses, which eventually bring the device back to a state close to its initial steady state, implying the potential for autonomic computing ${ }^{48,49}$.

The above PPF and PPD demonstrations were realized with diffusive memristors only, resembling short-term plasticity ${ }^{45}$ in synapses because any conductance change from the OFF state of diffusive memristors will vanish over time. When combined with a non-volatile element, i.e., a drift-type memristor, long-term plasticity ${ }^{50}$ following the spike-rate-dependent plasticity and $\mathrm{STDP}^{47,50}$ learning rules can be realized. For demonstration purposes, we created a combined circuit element using a diffusive memristor in series with a $\mathrm{Pt} / \mathrm{TaO}_{\mathrm{x}} / \mathrm{Ta} / \mathrm{Pt}$ drift memristor (Supplementary Fig. S8). This combined element was connected to pulsed voltage sources similar to a synapse between pre- and post-synaptic neurons (Fig. 6a). The spike-rate-dependent potentiation demonstration is illustrated in Fig. 6b, where the drift memristor weight (conductance) change is a function of the frequency of the applied pulses. Similar to Fig. 5b, a shorter $t_{\text {zero }}$ resulted in a greater increase in the conductance of the diffusive memristor and thus a larger voltage drop across the drift memristor, which thereby switched due to the voltage divider effect. A longer $t_{\text {zero }}$ resulted in a smaller increase in the diffusive memristor conductance and thus a smaller voltage drop across the drift memristor, leading to a smaller or non-detectable resistance change in the drift memristor. 
To demonstrate STDP learning rules with non-overlapping spikes, pre and post-synaptic spikes (Fig. 6c) were applied to the combined element. The two spikes were separated by a time difference $\Delta t$, which determined how much conductance change was programed in the drift memristor. Each spike consisted of two parts, a high voltage short pulse and a low voltage long pulse. The pre-spike and post-spike were equal in magnitude but opposite in voltage polarity (Fig. 6c). In the combined element, the resistance of the diffusive memristor in its OFF state is much larger than that of the drift memristor, while the resistance of its ON state is much smaller than that of the drift memristor. Because the diffusive memristor has a finite delay time, the short high voltage pulse will not turn it ON. In contrast, the long voltage pulse with a lower amplitude will turn ON the diffusive memristor. The drift memristor is not switched by the first spike, because the majority of the voltage drops across the diffusive memristor and turns it ON first. After the spike ends, the resistance of the diffusive memristor gradually increases from its ON state over time, regulated by the diffusive dynamics. The second spike occurs at a time $\Delta t$ from the end of the first spike, and it may or may not switch the drift memristor depending on how much voltage drops on the drift memristor, which is determined by the conductance of the diffusive memristor at that moment, a function of $\Delta t$. A smaller $\Delta t$ corresponds to a smaller diffusive memristor resistance and results in a greater resistance change in the drift memristor and vice versa (Fig. 6d). If the pre-spike appears before the post-spike, the drift memristor conductance increases (potentiation). If the prespike follows the post-spike, depression occurs. (Supplementary Fig. S9) Because the dynamics of the diffusive memristor provides an intrinsic timing mechanism for the combined element, the spike-rate-dependent plasticity and STDP do not require complex pulse engineering or spike overlapping. This substantially reduces the complexity of both circuit and algorithm design and enables low-energy operations. In addition, depending on 
the application, any non-volatile memristor (low/high retention, analog/digital) can be used along with the diffusive memristor, allowing a significantly broader choice of materials rather than relying on the properties of the drift memristor when used alone. (See Supplementary Fig. S10 for STDP demonstration with a Pt/HfO $/ \mathrm{TiN}$ drift memristor)

In conclusion, we have constructed and demonstrated a new class of memristors as synaptic emulators that function primarily based on diffusion (rather than drift) dynamics. The microscopic nature of both the threshold switching and relaxation of the diffusive memristor is revealed for the first time by in situ HRTEM and explained by nanoparticle dynamics simulation. The Ag dynamics of the diffusive memristors functionally resemble the synaptic $\mathrm{Ca}^{2+}$ behavior in chemical synapses and lead to a direct and natural emulation of multiple synaptic functions for both short-term and long-term plasticity, such as PPF, PPD, PPD following PPF, SRDP and STDP. In addition to providing a synapse emulator, the diffusive memristor can also serve as a selector with a large transient nonlinearity that is critical for the operation of a large crossbar array as a neural network. The results here provide an encouraging pathway toward synaptic emulation using diffusive memristors for neuromorphic computing.

\section{Methods}

Preparation of crossbar samples. The diffusive memristor devices were grown on p-type (100) Si wafer with $100 \mathrm{~nm}$ thermal oxide. The bottom electrodes were patterned by photolithography followed by evaporation and liftoff of a 20nm thick $\operatorname{Pt}(\mathrm{Au})$ layer. The $\sim 15 \mathrm{~nm}$ thick doped dielectric was deposited at room temperature by reactively co-sputtering MgO or $\mathrm{HfO}_{2}$ (99.99\%, Kurt Lesker) and Ag (99.99\%, Kurt Lesker) in an ambient of mixed Ar and $\mathrm{O}_{2}$, or co-sputtering Si (99.99\%, Kurt Lesker) and Ag in $\mathrm{Ar}, \mathrm{N}_{2}$, and $\mathrm{O}_{2}$. The $\sim 30 \mathrm{~nm}$ 
$\operatorname{Pt}(\mathrm{Au})$ top electrodes were subsequently patterned by photolithography followed by evaporation and liftoff processes. Electrical contact pads of the bottom electrodes were first patterned by photolithography and then subjected to reactive ion etching with mixed $\mathrm{CHF}_{3}$ and $\mathrm{O}_{2}$ gases.

The drift memristor devices used the same substrates and bottom electrodes as the diffusive memristor devices. The switching layer was grown by sputtering $\mathrm{Ta}_{2} \mathrm{O}_{5}\left(\mathrm{HfO}_{2}\right)$ for a thickness of $10(5) \mathrm{nm}$ in $\mathrm{Ar}$ gas followed by photolithography. Top electrodes were deposited by evaporating Ta(5nm)/Pt(20nm) (sputtering TiN(50nm)/Pd(30nm)) and liftoff.

Electrical Measurements. Electrical measurements were performed with the Keysight B1500A semiconductor device analyser using two of its modules: DC measurements were carried out using the source and measure units (B1517A) and the B1530A waveform generator/fast measurement unit (WGFMU) was used to perform the pulse measurements. Using a two-probe (W tips) configuration, we applied DC and pulse voltages between the top and bottom electrodes of the device and measured current through one of the measurement units. The same units were used to perform measurements at different temperatures on the Variable Temperature Micro Probe System (MMR technologies, K2000 Digital Temperature Controller) in ambient atmosphere. We performed the rate-dependent plasticity and the STDP experiments with the diffusive memristor in series with a $\mathrm{Pt} / \mathrm{TaO}_{\mathrm{x}} / \mathrm{Ta} / \mathrm{Pt}$ drift memristor. The B1530A WGFMU was used for applying the pre and spike-post-synaptic voltage spikes. The conductance of the drift memristor was read using a small DC voltage between each programming operation to determine the change in its weight. For each data point of the spike-rate-dependent plasticity measurement, the drift memristor was first initialized to its high resistance state, and then we applied 15 voltage pulses across the combined series memristors with the same pulse amplitude $(2.5 \mathrm{~V})$, duration $(40 \mu \mathrm{s})$ and a particular $t_{\text {zero }}$ value, 
and finallyread the drift memristor to determine the change in its state induced by the train of 15 pulses.

Preparation of planar samples for TEM. The specimen for in situ HRTEM was grown on the Aduro E-chip with pre-built Au connections (Model E-AEL00-LN, Protochip). Au electrodes are patterned by electron beam lithography followed by evaporation and liftoff of a $\sim 20 \mathrm{~nm}$ thick $\mathrm{Au}$ layer. The doped dielectric layer of $\mathrm{SiO}_{\mathrm{x}} \mathrm{N}_{\mathrm{y}}$ : $\mathrm{Ag}$ was deposited by reactively co-sputtering of both Si and Ag targets in an ambient of Ar, $\mathrm{N}_{2}$, and $\mathrm{O}_{2}$ at room temperature with a thickness of $\sim 30 \mathrm{~nm}$.

in situ TEM. The in situ HRTEM was performed at Brookhaven National Laboratory with the FEI Titan 80-300 operating at 300keV. The sample was grown on a Aduro E-chip (Model E-AEL00-LN, Protochip) which was mounted on a corresponding Aduro TEM holder for FEI (Protochip). Current was monitored in real-time on a Keithley 2602B System SourceMeter which exerted electrical bias.

\section{Diffusive memristor dynamical simulations.}

The diffusive memristor model links electrical, nano-mechanical and heat degrees of freedom: (a) the growth, shape change and decay of clusters of nanoparticles identified by their positions $x_{i}$ inside a device ( $i$ is the nanoparticle label), (b) the electric current through the device governed by a sequence of tunnelling resistances between nanoparticles, and (c) the local temperature controlling the nanoparticle diffusion, determined by Joule heating and the thermal conductivity of the memristor.

The mathematical definition for a generic memristor ${ }^{4}$ has two components, the quasi-static conduction equation relating voltage $v$ and current $i$, or Ohm's law for the element

$$
v=i R(\boldsymbol{x})
$$

where $R$ is the state-dependent resistance and $\boldsymbol{x}$ represents one or more state variables that define the physical properties of the memristor, and the dynamical equation(s) that define the 
evolution of the state variables with time in the presence of a current and affected by local temperature T:

$$
\frac{d x}{d t}=f(x ; i, T)
$$

For the state-dependent resistance we assume sequential electron tunnelling: first from the input terminal to the nearest metallic nanoparticle, and then from this nanoparticle to the next one and so on with the last tunnelling event to the output terminal. The total resistance of the memristor is the sum of tunnelling resistances between $N-1$ adjacent nanoparticles/islands:

$$
R_{M}=\sum_{0}^{N} \mathrm{R}_{t} \exp \left[\left(x_{i+1}-x_{i}\right) / \lambda\right]
$$

with $\lambda$ the effective tunnelling length, $\mathrm{R}_{t}$ the tunneling resistance amplitude (assumed the same for all islands), $x_{0}$ and $x_{N}$ the spatial coordinates of the input and output terminals, respectively, and we order the island positions as $x_{0}<x_{1}<x_{2}<\ldots<x_{N-1}<x_{N}$. The minimum resistance can be estimated as $R_{\min }=\min R_{M}=N R_{t} \exp [2 / N \lambda]$.

To describe the nanoparticle diffusion, and thus the memristor dynamics, we employed an over-damped Langevin equation for each mobile metallic nanoparticle trapped by a potential $U$ and subject to a random force $\xi_{i}$, the magnitude of which is determined by the device local temperature $T$,

$$
\eta \frac{d x_{i}}{d t}=-\frac{\partial U\left(x_{i}\right)}{\partial x_{i}}+\alpha \frac{V(t)}{L}+\sqrt{2 \eta k_{B} T} \xi_{i}
$$

In the equation (4) the friction term (left hand side), proportional to the particle velocity and viscosity $\eta$, is balanced by all other forces acting on nanoparticles. The first term on the right hand side of Eq. 4 forces the nanoparticle to approach the minimum potential energy with a speed that is proportional to minus the local gradient of the potential energy. The drift term $\alpha V(t) / L$ represents a bias in the electric filed $\mathrm{E}=V(t) / L$ affecting nanoparticle with induced 
charge $\alpha$ when the voltage is on. The electrical bias together with diffusion is responsible for switching to a high conducting state and depletion of nanoparticles at one of the device terminals. The random force, $\sqrt{2 \eta k_{B} T} \xi_{i}$, which is driven by the instantaneous temperature, describes diffusion of the nanoparticles that occurs both when voltage is on and when the system relaxes with no bias toward the potential minimum after the power to the device is off and the local temperature cools. In other words, since $T$ is nonzero, there will always be some diffusion of the nanoparticles modelled by adding random thermal fluctuations $\xi_{\text {i }}$, which are $\delta$-correlated white noise characterized by $\langle\xi\rangle=0$ and $\langle\xi(0) \xi(t)\rangle=\delta(t)$.

The potential $U=U_{I}+U_{p}$ has two energy scales - the interfacial energy $U_{I}$ responsible for formation of large metallic clusters near the device terminals and a weaker nanoparticlepinning energy $U_{p}$ with many smaller wells between the electrodes. Pinning can occur through interactions of the nanoparticles with impurities, the substrate and/or the ionic lattice $^{41}$. For our simulations we used $U_{I}=-w_{I}\left[\exp \left(-\frac{\left(x_{i}+x_{0}\right)^{2}}{R_{I}^{2}}\right)+\exp \left(-\frac{\left(x_{i}-x_{0}\right)^{2}}{R_{I}^{2}}\right)\right]$ and $U_{p}=\frac{w_{p}}{2} \sin \left(2 \pi \frac{x}{R_{p}}\right)$, with the interfacial energy barrier $w_{I}$ and the amplitude $w_{p}$ of the pinning potential. The particular shape of neither the interfacial potential nor the pinning energy profile are essential; the only property that matters is that the potential $U$ has two different energy scales and the weaker pinning potential has many wells (for example it can be random with fluctuating $w_{p}$ and $R_{p}$ ). At high enough temperature during a voltage pulse, the increased diffusion assisted by the electrical force breaks up the large nanoparticle clusters and populates the pinning sites. At lower temperatures after the power is off and the potential bias disappears, the nanoparticles re-aggregate to form clusters at the electrodes. Moreover, a qualitatively similar result (although requiring considerably more computational resources) has been obtained when we modelled the interfacial energy as an interaction between particles ${ }^{41}$. 
The temperature dynamics are determined by Newton's law of cooling:

$$
\frac{d T}{d t}=C_{T} Q-\kappa\left(T-T_{0}\right)
$$

with the Joule heating power $Q=V^{2} / R_{M}$ increasing the temperature of the system and thermal conductivity acting as a damping factor by removing energy from the system. Here, $\kappa$ and $C_{T}$ are the heat transfer coefficient and heat capacitance respectively, while the background temperature is $T_{0}$. Equations (4) and (5) are sufficient to describe the memristor dynamics subject to the current through the device, which is controlled by the electric circuit in which it is embedded. Thus, the model described above enables the simulation of a complex system consisting of both volatile and non-volatile memristors.

There are two time scales in the model, the diffusion time scale and the characteristic scale of temperature relaxation. The first one depends on temperature, potential profile, and electric drift, thus, this time scale changes depending on voltage pulse intensity and temperature. For this reason, we measure all time in the simulations in units of the temperature relaxation time $1 / \kappa$. As one can see from Fig. 4, the relaxation occurs on time scales of the order $10-201 / \kappa$, thus, the simulated system is clearly governed by diffusion rather than the temperature relaxation ${ }^{19}$. There are two conductance states with low and high conductance. We used the conductance $1 / R_{\min }$ in the high conductance state as a normalization factor. The normalization enabled the simulated conductance to be plotted as a percentage of its maximum conductance after each applied pulse, for instance, from Fig $4 \mathrm{~b}$ we conclude that the conductance reached $25 \%$ of the maximum after 4 pulses and about $75 \%$ after 7 pulses. To provide a better link to the experimental data, we normalized the voltages to the threshold voltage $V_{t h}$, which was assumed to be the voltage where the conductance reached $10 \%$ of its maximum value. These quantities can be easily obtained from both simulations (averaged over 30 realizations) and experimental I-V hysteresis loops, thus directly linking the units in 
the simulations and the experiments. For the sake of consistency, we normalized all voltages to $V_{t h}$ at the highest simulated temperature, $\frac{k_{B} T}{w_{p}}=0.45$. The temperature $\frac{k_{B} T}{w_{p}}$ in the simulations was varied from 0.3 to 0.45 (i.e, the same relative range as in Fig. 3b). In this range the characteristic single hopping time scale $e^{w_{p} / k_{B} T}$ changed from about $2 / \kappa$ to $6 / \kappa$ and the diffusion relaxation time (estimated as a characteristic jump time multiplied by the number of jumps to reach the interfacial minimum near the device terminal) was about $20 / \kappa$ to $60 / \kappa$. The ratio of interfacial and pinning potentials $w_{I} / w_{p}$ was assumed to be 4.5 in the simulations to ensure that the interfacial potential was strong enough to re-trap the $\mathrm{Ag}$ nanoparticles after the voltage was switched off and the thermal fluctuations were too weak to destroy the large clusters near the terminals in the absence of excess heating. Conversely, the electric force ( $\alpha V_{t h} R_{p} / w_{I}=0.3$ in the simulations) was used to suppress the interfacial barrier $k_{B} w_{I} / T$ to about 3.6, which enabled the enhanced thermal fluctuations induced by Joule heating to activate the positive thermal feedback. The stronger fluctuations support larger Joule heating and vice versa, breaking up the large Ag clusters to form a string of smaller nanoparticles and thus a conductive path between the terminals, as observed experimentally in the HRTEM studies. Note that we also linked some model parameters with experimentally measurable ones, e.g., $C_{T} \approx \kappa w_{p} R_{t} / V_{t h}^{2} k_{B}, \eta \approx w_{p} / \kappa L^{2}$, and $\alpha \approx \eta \kappa L^{2} / V_{t h}$. 


\section{References}

1 Diorio, C., Hasler, P., Minch, B. A. \& Mead, C. A. A single-transistor silicon synapse. IEEE Trans. Electron Dev. 43, 1972-1980, (1996).

2 Indiveri, G., Chicca, E. \& Douglas, R. A VLSI array of low-power spiking neurons and bistable synapses with spike-timing dependent plasticity. IEEE Trans. Neu. Net. 17, 211-221, (2006).

3 Bartolozzi, C. \& Indiveri, G. Synaptic Dynamics in Analog VLSI. Neural Comput. 19, 2581-2603, (2007).

4 Chua, L. Memristor-The missing circuit element. IEEE Trans. Circuit Theory 18, 507-519, (1971).

5 Terabe, K., Hasegawa, T., Nakayama, T. \& Aono, M. Quantized conductance atomic switch. Nature 433, 47-50, (2005).

6 Waser, R. \& Aono, M. Nanoionics-based resistive switching memories. Nat. Mater. 6, 833-840, (2007).

7 Kwon, D.-H. et al. Atomic structure of conducting nanofilaments in $\mathrm{TiO}_{2}$ resistive switching memory. Nat. Nanotechnol. 5, 148-153, (2010).

8 Wedig, A. et al. Nanoscale cation motion in TaOx, HfOx and TiOx memristive systems. Nat. Nanotechnol. 11, 67-74, (2016).

9 Jo, S. H. et al. Nanoscale Memristor Device as Synapse in Neuromorphic Systems. Nano Lett. 10, 1297-1301, (2010).

10 Yu, S., Wu, Y., Jeyasingh, R., Kuzum, D. \& Wong, H. S. P. An Electronic Synapse Device Based on Metal Oxide Resistive Switching Memory for Neuromorphic Computation. IEEE Trans. Electron Devices 58, 2729-2737, (2011).

11 Ohno, T. et al. Short-term plasticity and long-term potentiation mimicked in single inorganic synapses. Nat. Mater. 10, 591-595, (2011).

12 Wang, Z. Q. et al. Synaptic Learning and Memory Functions Achieved Using Oxygen Ion Migration/Diffusion in an Amorphous InGaZnO Memristor. Adv. Funct. Mater. 22, 2759-2765, (2012).

13 Lim, H., Kim, I., Kim, J. S., Hwang, C. S. \& Jeong, D. S. Short-term memory of $\mathrm{TiO}_{2}$-based electrochemical capacitors: empirical analysis with adoption of a sliding threshold. Nanotechnology 24, 384005, (2013).

14 La Barbera, S., Vuillaume, D. \& Alibart, F. Filamentary Switching: Synaptic Plasticity through Device Volatility. ACS Nano 9, 941-949, (2015).

15 Prezioso, M. et al. Training and operation of an integrated neuromorphic network based on metal-oxide memristors. Nature 521, 61-64, (2015).

16 Pershin, Y. V. \& Di Ventra, M. Experimental demonstration of associative memory with memristive neural networks. Neural Netw. 23, 881-886, (2010).

17 Pershin, Y. V. \& Di Ventra, M. Practical Approach to Programmable Analog Circuits With Memristors. IEEE Trans. Circuits Syst. I 57, 1857-1864, (2010).

18 Pershin, Y. V. \& Di Ventra, M. Memristive circuits simulate memcapacitors and meminductors. Electron. Lett. 46, 517-518, (2010).

19 Kim, S. et al. Experimental Demonstration of a Second-Order Memristor and Its Ability to Biorealistically Implement Synaptic Plasticity. Nano Lett. 15, 2203-2211, (2015).

20 Du, C., Ma, W., Chang, T., Sheridan, P. \& Lu, W. D. Biorealistic Implementation of Synaptic Functions with Oxide Memristors through Internal Ionic Dynamics. Adv. Funct. Mater. 25, 4290-4299, (2015). 
21 Xu, Z., Bando, Y., Wang, W., Bai, X. \& Golberg, D. Real-Time In Situ HRTEMResolved Resistance Switching of $\mathrm{Ag}_{2} \mathrm{~S}$ Nanoscale Ionic Conductor. ACS Nano 4, 2515-2522, (2010).

22 Liu, Q. et al. Real-time observation on dynamic growth/dissolution of conductive filaments in oxide-electrolyte-based ReRAM. Adv. Mater. 24, 1844-1849, (2012).

23 Yang, Y. et al. Observation of conducting filament growth in nanoscale resistive memories. Nat. Commun. 3, 732, (2012).

24 Yang, Y. et al. Electrochemical dynamics of nanoscale metallic inclusions in dielectrics. Nat. Commun. 5, 4232, (2014).

25 Tian, X. et al. Bipolar electrochemical mechanism for mass transfer in nanoionic resistive memories. Adv. Mater. 26, 3649-3654, (2014).

26 Hubbard, W. A. et al. Nanofilament Formation and Regeneration During $\mathrm{Cu} / \mathrm{Al}_{2} \mathrm{O}_{3}$ Resistive Memory Switching. Nano Lett. 15, 3983-3987, (2015).

27 Jo, S. H., Kumar, T., Narayanan, S., Lu, W. D. \& Nazarian, H. in Electron Devices Meeting (IEDM), 2014 IEEE International. 6.7.1-6.7.4.

28 Song, J., Woo, J., Prakash, A., Lee, D. \& Hwang, H. Threshold Selector with High Selectivity and Steep Slope for Cross-Point Memory Array. IEEE Electron Device Lett. 36, 681-683, (2015).

29 Yang, H. et al. in VLSI Technology (VLSI Technology), 2015 Symposium on. T130T131.

30 Luo, Q. et al. in Electron Devices Meeting (IEDM), 2015 IEEE International 10.14.11-10.14.14 (2015).

31 Voorhees, P. W. The theory of Ostwald ripening. J. Stat. Phys. 38, 231-252, (1985).

32 Stoneham, A. M. Systematics of metal-insulator interfacial energies: A new rule for wetting and strong catalyst-support interactions. Appl. Surf. Sci. 14, 249-259, (1983).

33 Sun, J. et al. Liquid-like pseudoelasticity of sub-10-nm crystalline silver particles. Nat. Mater. 13, 1007-1012, (2014).

34 Valov, I. \& Staikov, G. Nucleation and growth phenomena in nanosized electrochemical systems for resistive switching memories. J. Solid State Electrochem. 17, 365-371, (2012).

35 van den Hurk, J., Linn, E., Zhang, H., Waser, R. \& Valov, I. Volatile resistance states in electrochemical metallization cells enabling non-destructive readout of complementary resistive switches. Nanotechnology 25, 425202, (2014).

36 Guzman, D. M., Onofrio, N. \& Strachan, A. Stability and migration of small copper clusters in amorphous dielectrics. J. Appl. Phys. 117, 195702, (2015).

37 Hasegawa, T., Terabe, K., Tsuruoka, T. \& Aono, M. Atomic switch: atom/ion movement controlled devices for beyond von-Neumann computers. Adv. Mater. 24, 252-267, (2012).

38 Valov, I. et al. Atomically controlled electrochemical nucleation at superionic solid electrolyte surfaces. Nat. Mater. 11, 530-535, (2012).

39 Tsuruoka, T. et al. Effects of Moisture on the Switching Characteristics of OxideBased, Gapless-Type Atomic Switches. Adv. Funct. Mater. 22, 70-77, (2012).

40 Valov, I. \& Lu, W. D. Nanoscale electrochemistry using dielectric thin films as solid electrolytes. Nanoscale, (2016).

$41 \mathrm{Yi}, \mathrm{W}$. et al. Quantized conductance coincides with state instability and excess noise in tantalum oxide memristors. Nat. Commun. 7, 11142, (2016).

42 Burgoyne, R. D. Neuronal calcium sensor proteins: generating diversity in neuronal $\mathrm{Ca}^{2+}$ signalling. Nat. Rev. Neurosci. 8, 182-193, (2007).

43 Clapham, D. E. Calcium signaling. Cell 131, 1047-1058, (2007). 
44 Catterall, W. A. \& Few, A. P. Calcium channel regulation and presynaptic plasticity. Neuron 59, 882-901, (2008).

45 Zucker, R. S. \& Regehr, W. G. Short-Term Synaptic Plasticity. Annu. Rev. Physiol. 64, 355-405, (2002).

46 Malenka, R. C. \& Bear, M. F. LTP and LTD: An Embarrassment of Riches. Neuron 44, 5-21, (2004).

47 Caporale, N. \& Dan, Y. Spike timing-dependent plasticity: a Hebbian learning rule. Annu Rev Neurosci 31, 25-46, (2008).

48 Feng, L., Molnár, P. \& Nadler, J. V. Short-Term Frequency-Dependent Plasticity at Recurrent Mossy Fiber Synapses of the Epileptic Brain. J. Neurosci. 23, 5381-5390, (2003).

49 Mulkey, R., Herron, C. \& Malenka, R. An essential role for protein phosphatases in hippocampal long-term depression. Science 261, 1051-1055, (1993).

50 Bi, G.-q. \& Poo, M.-m. Synaptic Modifications in Cultured Hippocampal Neurons: Dependence on Spike Timing, Synaptic Strength, and Postsynaptic Cell Type. J. Neurosci. 18, 10464-10472, (1998). 


\section{Acknowledgements}

This work was supported in part by the U.S. Air Force Research Laboratory (AFRL) (Grant No. FA8750-15-2-0044), the Intelligence Advanced Research Projects Activity (IARPA) (contract 2014-14080800008), U.S. Air Force Office for Scientific Research (AFOSR) (Grant No. FA9550-12-1-0038), and the National Science Foundation (NSF) (ECCS-1253073). Any opinions, findings and conclusions or recommendations expressed in this material are those of the authors and do not necessarily reflect the views of AFRL. Part of the device fabrication was conducted in the clean room of the Center for Hierarchical Manufacturing (CHM), an NSF Nanoscale Science and Engineering Center (NSEC) located at the University of Massachusetts Amherst. The TEM work used resources of the Center for Functional Nanomaterials, which is a U.S. DOE Office of Science Facility, at Brookhaven National Laboratory under Contract No. DE-SC0012704. The authors thank Mark McLean for useful discussions on computing.

\section{Author contributions:}

J.J.Y. conceived the concept. J.J.Y., Q.X., Z.W. and S.J. designed the experiments. Z.W. fabricated the devices and S.J. performed electrical measurements. S.E.S. performed the simulation. H.L.X. carried out the in-situ TEM characterizations. H.J., R.M., P.L., M.H., N.G., J.P.S., Z.L., Q.W., M.B., G.-L.L., and R.S.W. helped with experiments and data analysis. J.J.Y., Q.X., Z.W., S.J., S.E.S., and R.S.W. wrote the paper. All authors discussed the results and implications and commented on the manuscript at all stages.

\section{Additional information}


Supplementary information is available in the online version of the paper. Reprints and permissions information is available online at www.nature.com/reprints. Correspondence and requests for materials should be addressed to J.J.Y.

\section{Competing financial interests}

The authors declare no competing financial interests.

\section{Figure Captions}

Figure 1 Highly non-linear, fast, and repeatable threshold switching behaviours of diffusive memristors (a) Pseudo-colour scanning electron micrograph of a crossbar device. Top electrodes are depicted by the red dashed line and bottom contacts by the blue dashed line. Biasing is applied on the top electrode with the bottom electrode grounded. The inset shows an atomic force microscopy (AFM) image of the junction. (b) Repeatable highly non-linear threshold switching I-V loops for devices with different host lattices doped with silver. (c) High-speed switching characteristics of the $\mathrm{SiO}_{\mathrm{x}} \mathrm{N}_{\mathrm{y}}$ : $\mathrm{Ag}$ device at an elevated temperature of $600 \mathrm{~K}$. The programming pulse width is $500 \mathrm{~ns}$ and the voltage is $1.5 \mathrm{~V}$. (d) Endurancecycling performance test of the $\mathrm{SiO}_{\mathrm{x}} \mathrm{N}_{\mathrm{y}}$ :Ag device for 1 million cycles at room temperature. The inset shows the shape of the applied voltage pulse, which consists of four segments: a $1.4 \mathrm{~V} / 200 \mu \mathrm{s}$ programming voltage and a $0.14 \mathrm{~V} / 200 \mu \mathrm{s}$ read voltage, followed by a $1.4 \mathrm{~V} / 200 \mu \mathrm{s}$ programming voltage and a $-0.14 \mathrm{~V} / 200 \mu$ s read voltage. We sampled one voltage and one current data point from each of the four segments of the pulse. The voltage samples are shown in red, while the purple dots and green dots correspond to device current due to positive and negative programming pulses, respectively. The read current for both positive and negative programming is at the instrument noise level $(<100 \mathrm{nA})$ for the current 
measurement range used. The device did not fail during the measurement, demonstrating high robustness.

Figure 2 In situ TEM observation of the threshold switching process suggesting the relaxation is a diffusion process driven by interfacial energy minimization. External electric field is exerted at the point of time zero. Ag migration is observed at time $0.1 \mathrm{~s}$ when two nanocrystals started to form. A clear arc-shaped filament is visible at 2.5s. When the external biasing is removed at $5.0 \mathrm{~s}$, the filament starts to deform, shrinking to a round spherical nanocluster implying an interfacial energy driven diffusion mechanism. All scale bars, 20nm. Figure 3 Timing mechanism of $\mathrm{SiO}_{\mathrm{x}} \mathrm{N}_{\mathrm{y}}$ :Ag diffusive memristor. (a) Delay and relaxation characteristics of the device showing variation of current (blue) with applied voltage (red) pulses. Multiple read voltage pulses of $(0.05 \mathrm{~V}, 10 \mu \mathrm{s})$ are used to study the device relaxation current after the switching pulse $(0.75 \mathrm{~V}, 5 \mathrm{~ms})$. The device requires a finite delay time to turn ON and has a finite relaxation time before it goes to the high resistance state after the switching pulse is removed. (b) Device relaxation performance showing the variation of current with applied voltage at different temperatures. The relaxation time decreases with increasing temperature. Inset shows the Arrhenius plot of the temperature dependence of the relaxation time. Each data point (black circles) is an average over 10-15 measured relaxation times, and are fitted to the blue line. The activation energy for the material system is calculated to be $0.27 \mathrm{eV}$.

Figure 4 Simulated operation of a diffusive memristor device. (a) The conductance response (blue curve) induced by a voltage pulse (red curve) shows a delay in the response and a gradual relaxation towards the low conducting state, consistent with the experimental threshold switching property in Fig. 3a. Panels (a1-a4) show evolution of metallic nanoparticle density distributions. Blue curves represent particle distributions. Each curve was averaged over temporal interval $0.2 / \kappa$ around time moments indicated by orange dots (in (a)) when 
instantaneous particle snapshots (red dots) are plotted. The inset shows the temperature dependence of the relaxation time $\tau$ defined as the time needed for the conductance to drop to value $0.02 G_{\max }$ after switching the voltage, which agrees with that of Fig. 3b. The red curve is the fitting of $\ln \left(\kappa \tau_{r}\right)$ by the function $A+B w_{p} / k_{B} T$ with $A=0.1, B=1$. (b) The simulated conductance response (blue curve) showing both facilitation and then depression upon a sequence of short and frequent pulses (red curve). Panels (b1-b6) show evolution of metallic nanoparticle density. Blue curves represent particle distributions, each curve was averaged over temporal interval $0.02 / \kappa$, around time moments indicated by orange dots (in (b)) when instantaneous particle snapshots (red dots) are plotted. The conductance initially increases with number of pulses until a conducting-bridge between terminals is formed. The conductance eventually saturates and drops as the number of pulses increase further, which captures an advanced synaptic behaviour, i.e., PPF followed by PPD. Other simulation parameters are $\lambda / 2=R_{I}=2 R_{p} / 3=L / 10, x_{0}=0.85 L$, we simulate a hundred of nanometallic particles and the simulation step was $d t=2 \times 10^{-6}$ resulting in $10^{7}$ steps during each simulation run; the reasoning of parameters choice and pinning parameters are discussed in the method section.

Figure 5 Schematic illustration of the analogy between $\mathrm{Ca}^{2+}$ and $\mathrm{Ag}$ dynamics, and short term synaptic plasticity of the diffusive memristor. (a) (left) Diffusion of $\mathrm{Ca}^{2+}$ from extracellular sources via VSCC and NMDAR, and the removal of $\mathrm{Ca}^{2+}$ via PMCA and NCE. (right) Ag diffusion into the gap region between Ag nanoclusters with field assistance and clearance of Ag filament from the gap region by the interfacial energy or mechanical stress once the voltage signal is removed, which is a close emulation of $\mathrm{Ca}^{2+}$ dynamics. (b) Experimental demonstration of short-term synaptic paired-pulse facilitation (PPF) and depression (PPD) behaviour with the diffusive $\mathrm{SiO}_{\mathrm{x}} \mathrm{N}_{\mathrm{y}}$ : $\mathrm{Ag}$ memristor. Device current response (blue) to multiple subsequent voltage pulses (3V, 1ms). The duration between two 
pulses when the applied voltage is $0 \mathrm{~V}$ is denoted as $t_{\text {zero }}$. For small $t_{\text {zero }}$, the current increases with the number of pulses giving larger conductance (weight) change, demonstrating pairedpulse facilitation. Inset shows the percentage change in conductance (weight) for different $t_{\text {zero }}$ values. As the $t_{\text {zero }}$ increases from $1 \mathrm{~ms}$ to $160 \mathrm{~ms}$, the weight change slows down with increasing pulse number and eventually becomes negative. (c) Experimental demonstration of PPD following PPF in the diffusive $\mathrm{SiO}_{\mathrm{x}} \mathrm{N}_{\mathrm{y}}$ :Ag memristor. Device current (blue) response to a train of voltage pulses $(2.8 \mathrm{~V}, 100 \mu \mathrm{s})$ of the same amplitude but different frequencies. The device begins with PPD (depression) upon low frequency $(196 \mathrm{~Hz})$ stimulation and experiences an increase in current (facilitation) once the stimulation frequency is raised $(5000 \mathrm{~Hz})$. The brief facilitation is followed by depressed current under identical stimulation frequency $(5000 \mathrm{~Hz})$ but excessive pulse number, as a result of the gradual depletion of silver at one electrode and accumulation at the other. The current relaxes to its initial value with subsequent low frequency stimulation $(196 \mathrm{~Hz})$, implying potential for autonomic computing $^{48,49}$.

Figure 6 Long-term spike-rate-dependent potentiation and bio-realistic spike-timing dependent plasticity (SRDP and true STDP) behaviour of a combined device consisting of a diffusive and a drift memristor. (a) Illustration of a biological synaptic junction between the pre- and post-synaptic neurons. Also shown is the electrical implementation, a circuit diagram of the electronic synapse consisting of the $\mathrm{SiO}_{\mathrm{x}} \mathrm{N}_{\mathrm{y}}$ : Ag diffusive memristor connected in series with the $\mathrm{TaO}_{\mathrm{x}}$ drift memristor and between pulsed voltage sources, which act as neurons that send voltage spikes to the synaptic junction. (b) Spike-rate-dependent potentiation showing the change in the conductance (weight) of the drift memristor in the electronic synapse with change in the duration $t_{\text {zero }}$ between the applied pulses. For long $t_{\text {zero, }}$ the change in the conductance of the diffusive memristor is lower (see Fig. 5b), resulting in a lower weight change of the drift memristor. As the $t_{\text {zero }}$ decreases, the weight change 
increases. The dotted red line represents a fit of the average conductance change with change in $t_{\text {zero }}$ (c) Schematic of the pulses applied to the combined device for STDP demonstration. The long low voltage pulse in each spike turns the diffusive memristor ON, and the short high voltage pulse switches the drift memristor. When the post-spike precedes the pre-spike, the device is reset (depressed), and when the pre-spike precedes the post-spike, the device is set (potentiated). The timing $(\Delta t)$ between the two spikes determines the voltage drop across the drift memristor. (d) Plot of the conductance (weight) change of the drift memristor with variation in $\Delta t$ showing the spike-timing dependent plasticity of the electronic synapse. This response is characteristic of the timing-dependent response of biological synapses. The inset shows the spike-timing-dependent plasticity of a typical chemical synapse (reprinted from Fig. 7 of Ref. 50). 

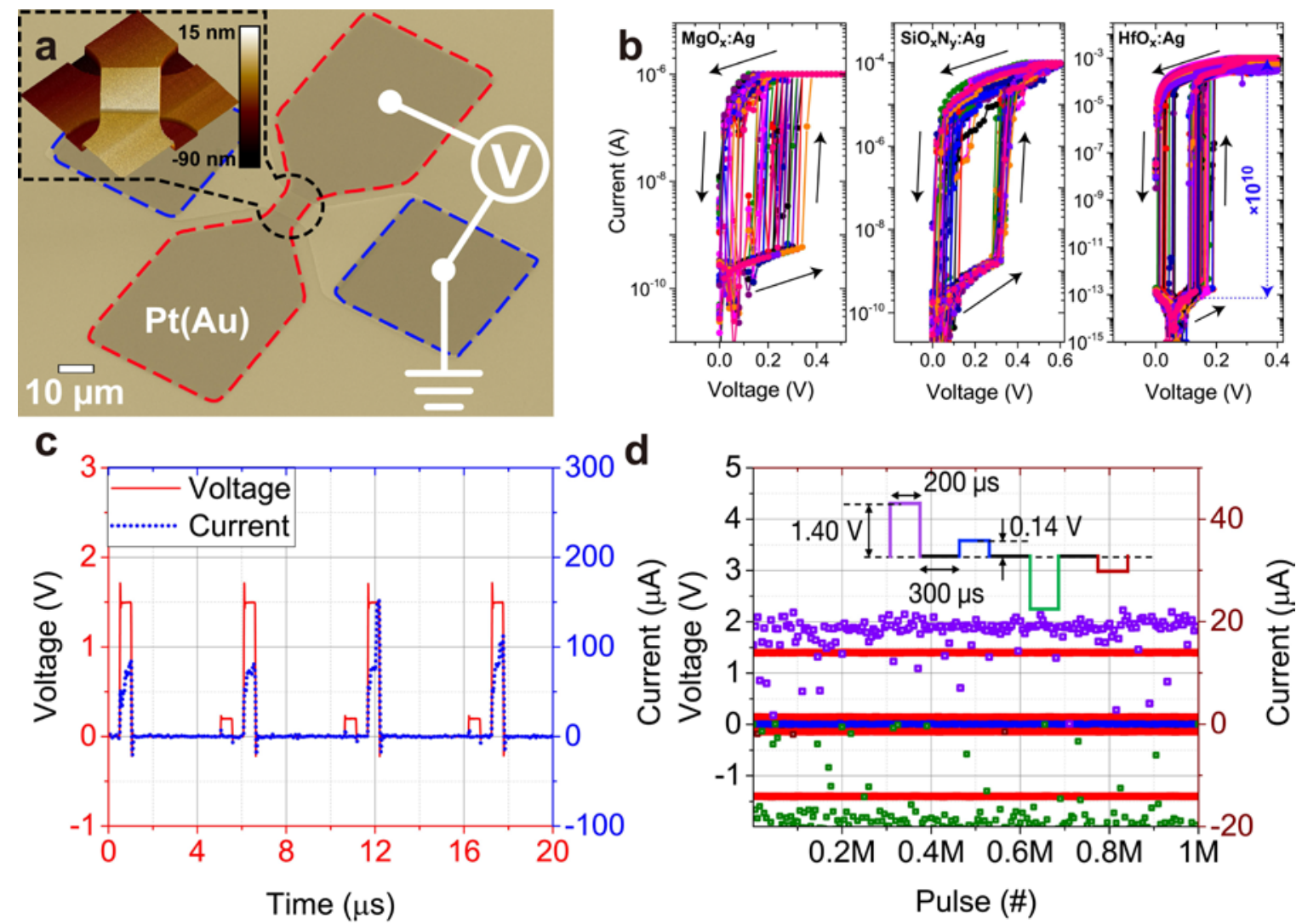

Figure 1 


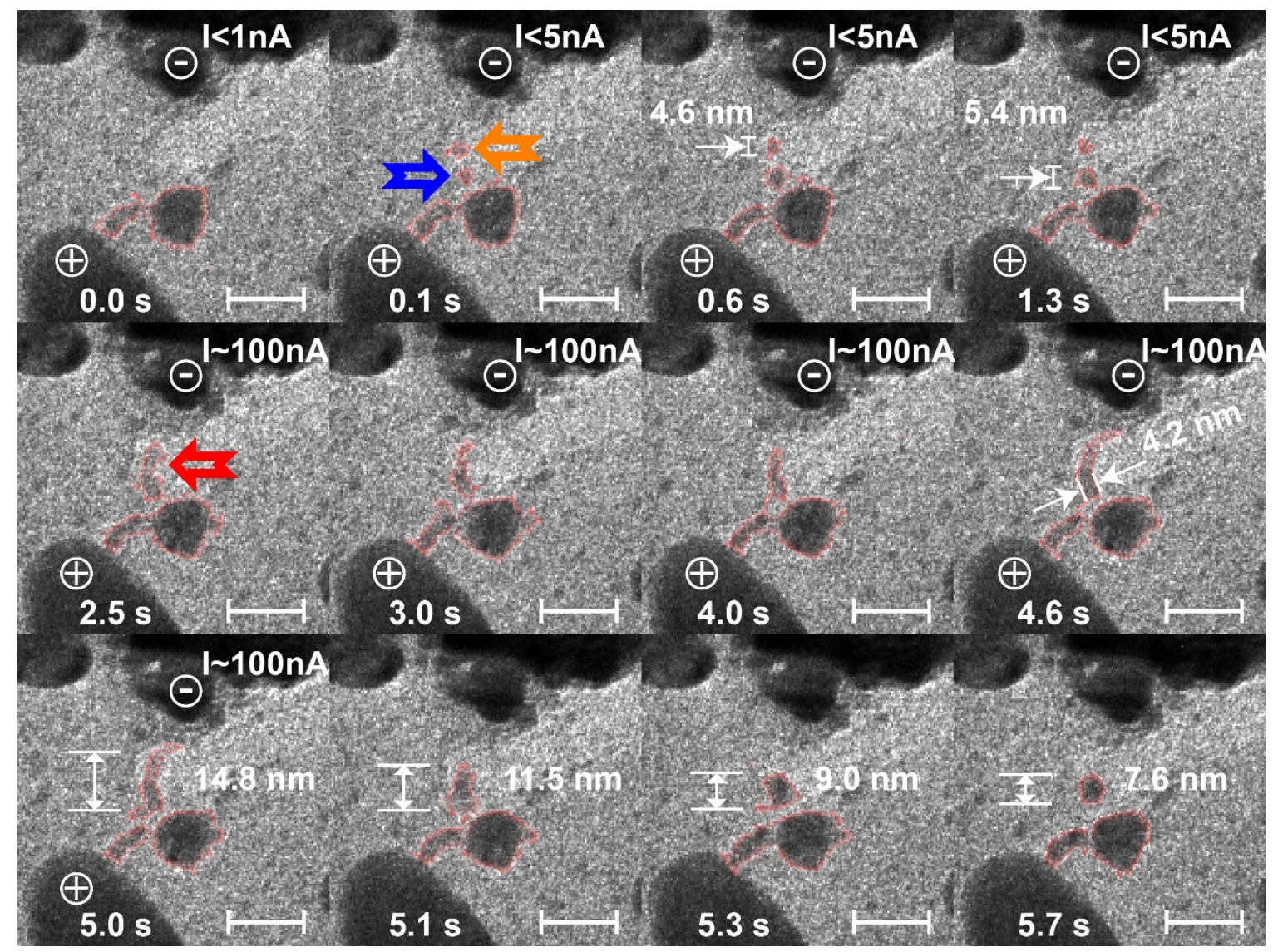

Figure 2 

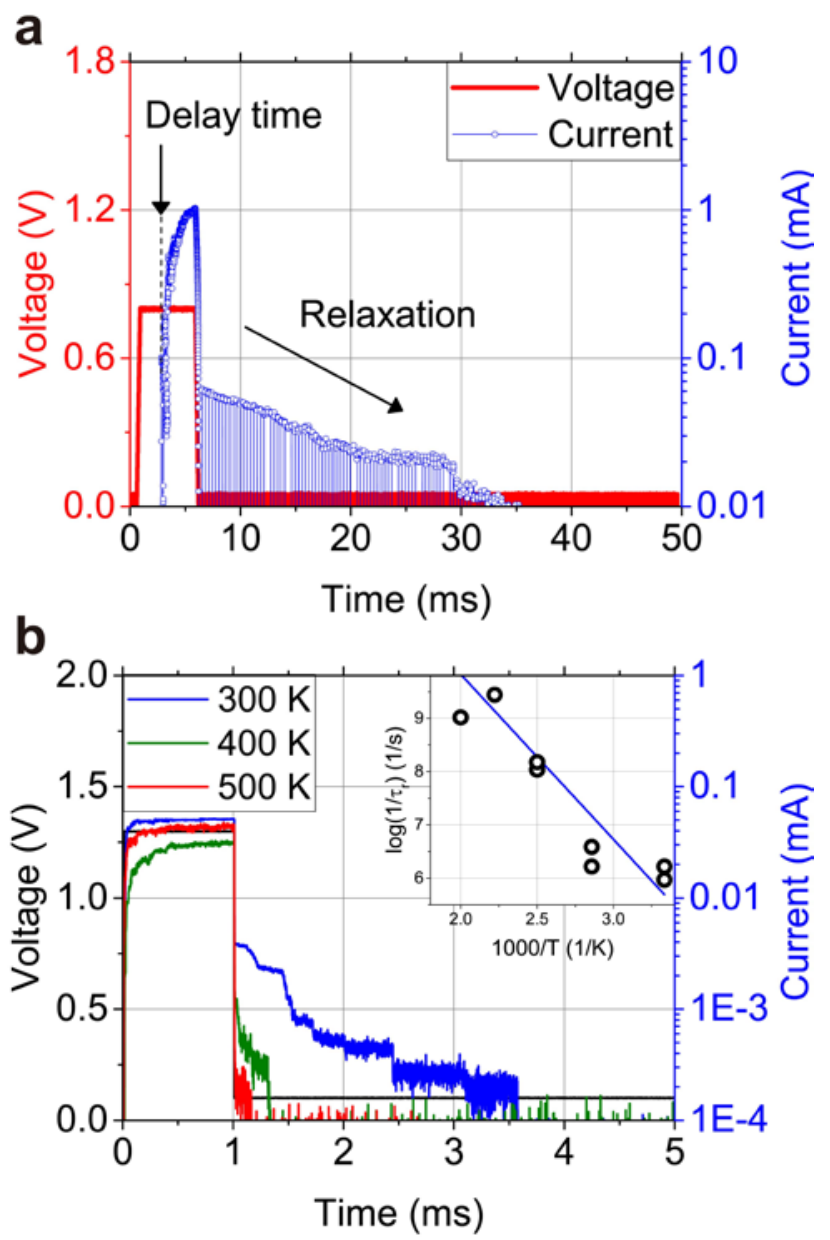

Figure 3 

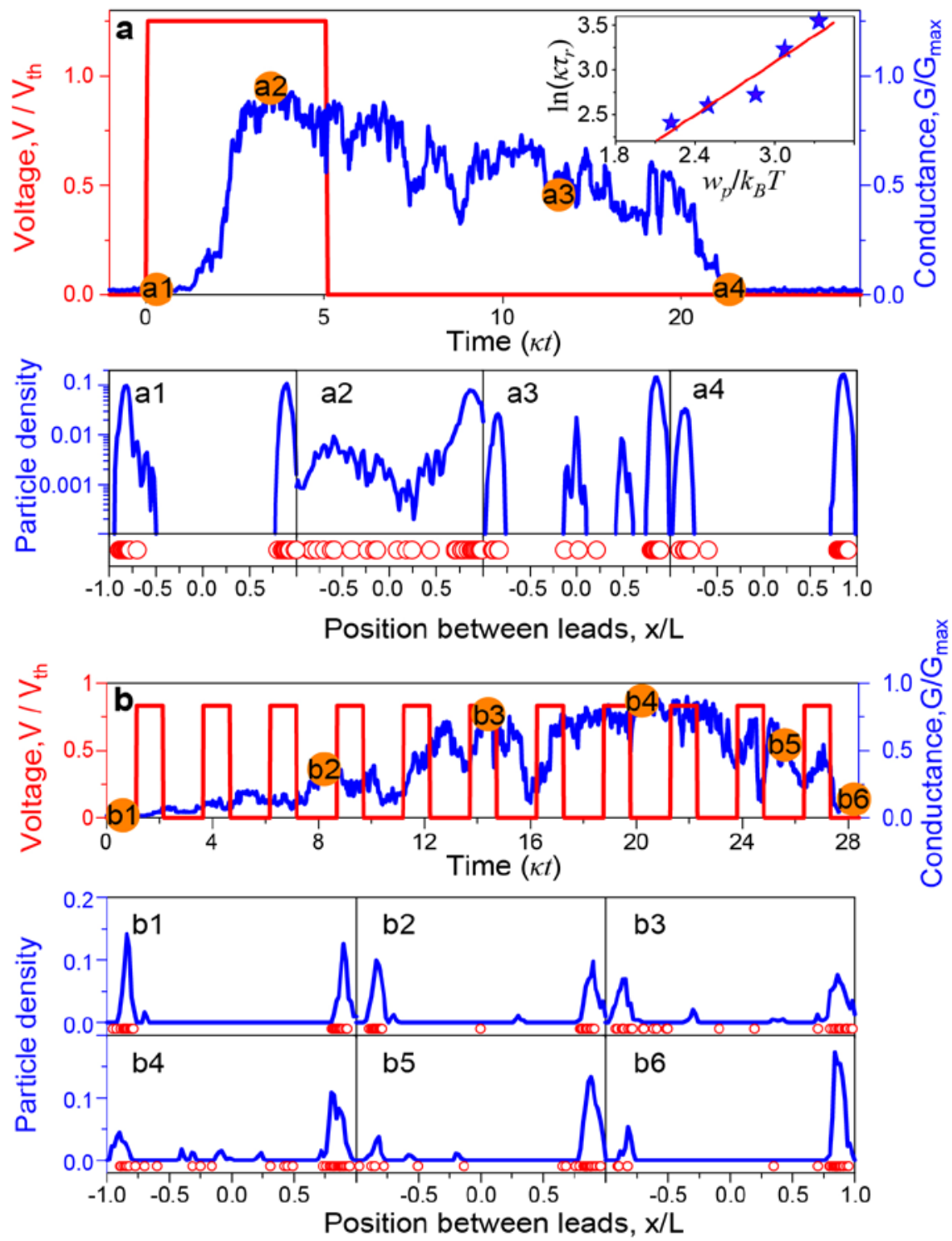

Figure 4 


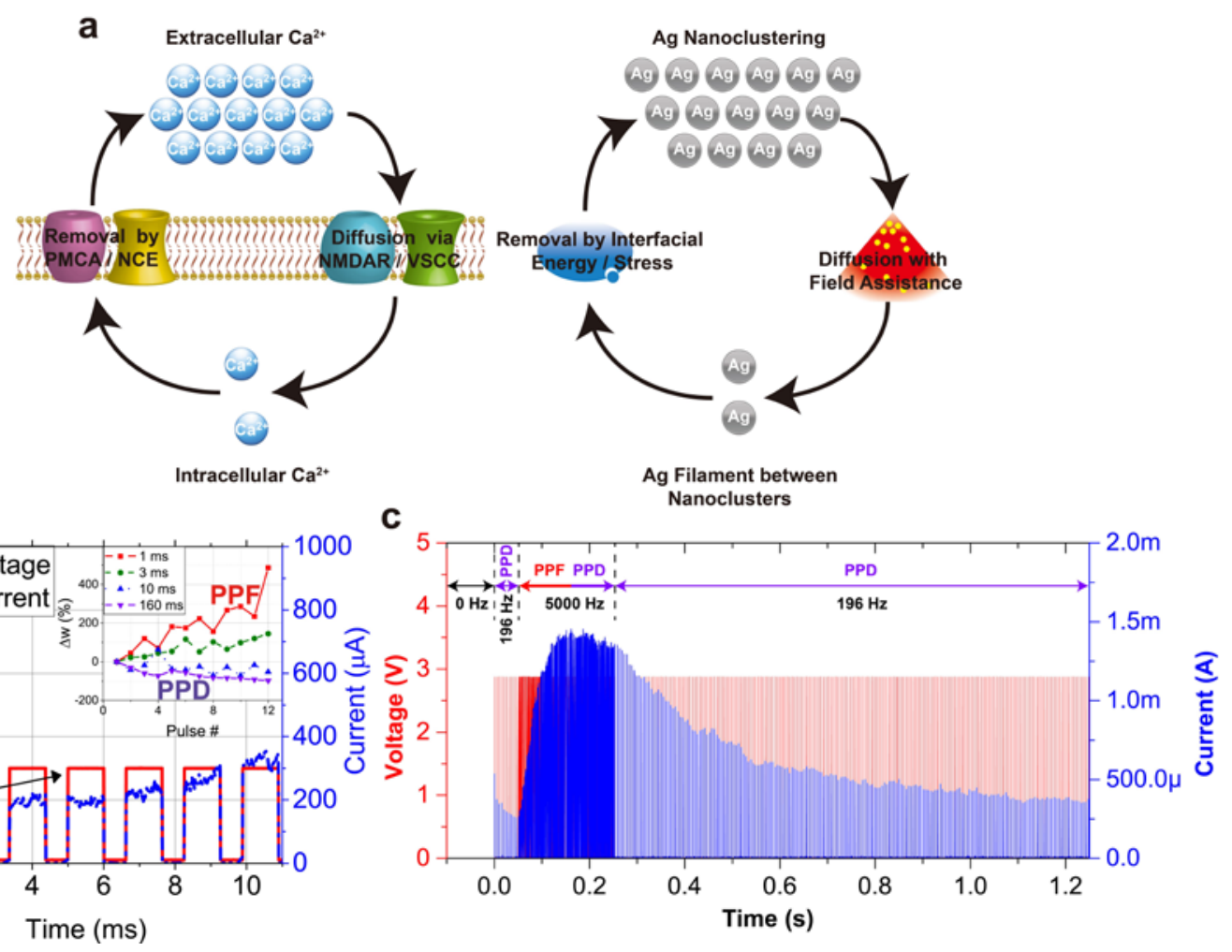

Figure 5 

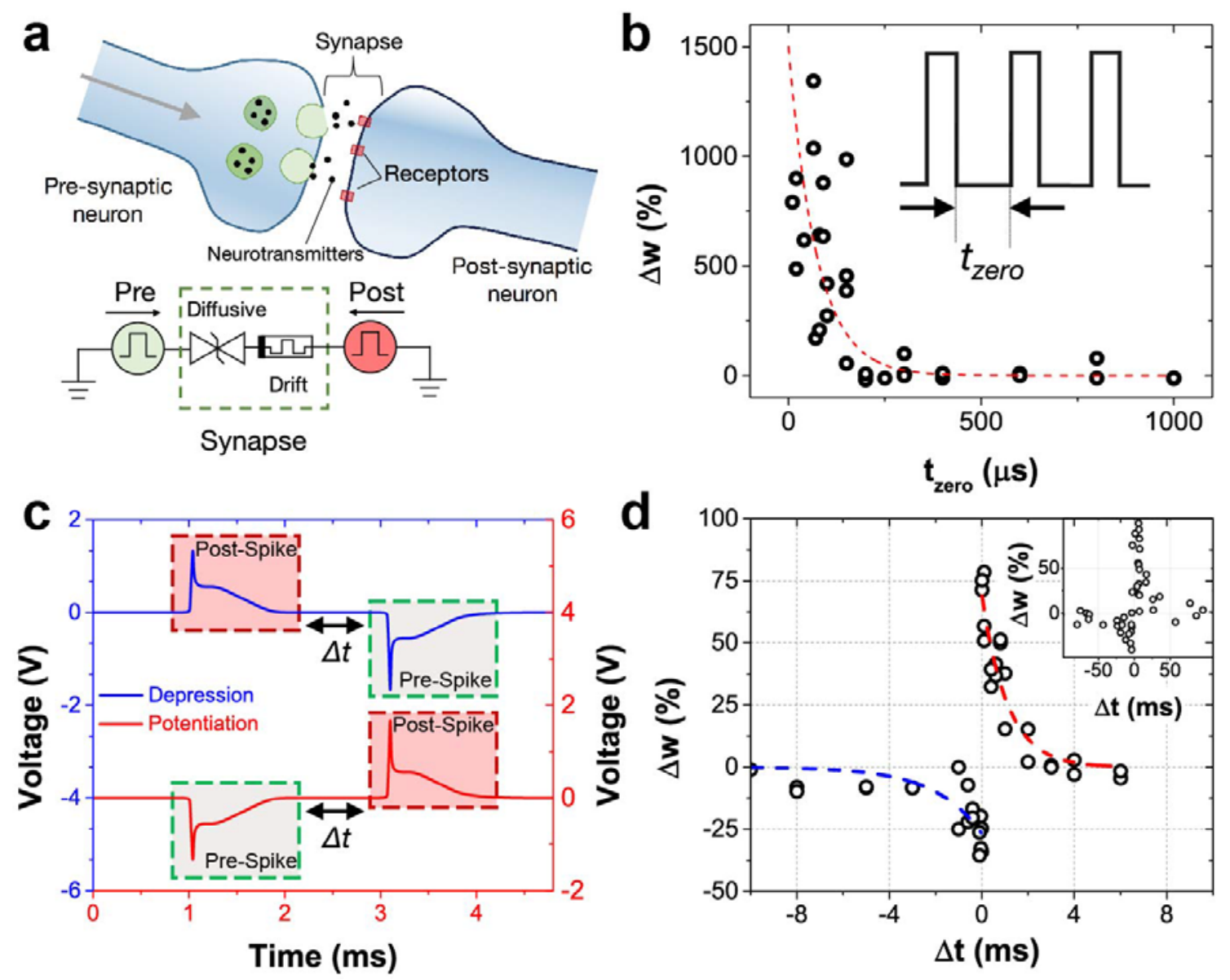

Figure 6 

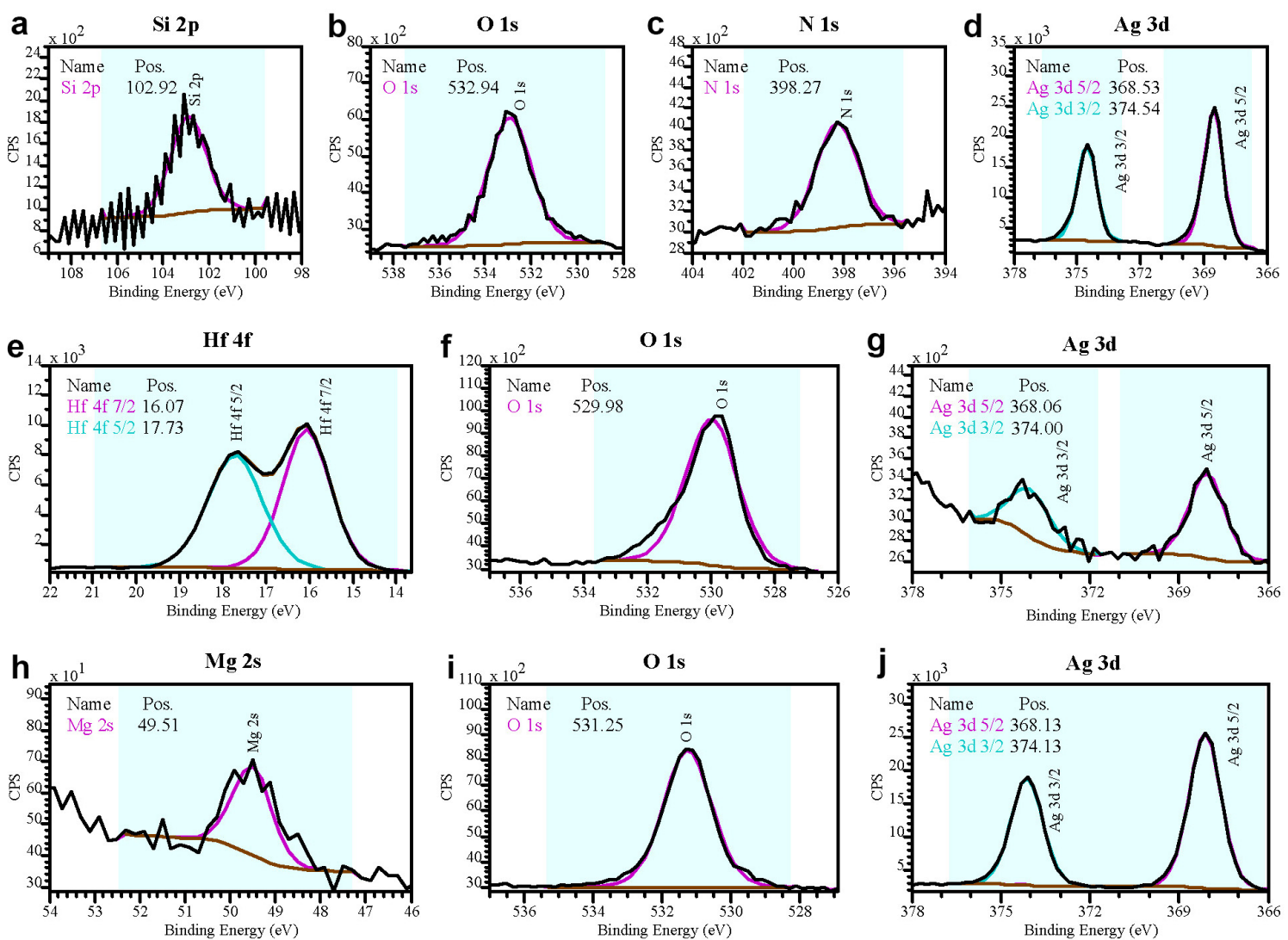

Supplementary Figure S1 X-ray Photoelectron Spectroscopy (XPS) of (a-d) $\mathrm{SiO}_{x} \mathrm{~N}_{\mathrm{y}} \mathrm{Ag}$ (e-

g) $\mathrm{HfO}_{\mathrm{x}}: \mathrm{Ag}$, and (h-j) $\mathrm{MgO}_{\mathrm{x}}: \mathrm{Ag}$ films. All spectrums are calibrated by align $\mathrm{C} 1 \mathrm{~s}$ to 284.6 $\mathrm{eV}$. The binding energy of $\mathrm{Ag}$ in all three systems indicate a metallic nature of majority $\mathrm{Ag}$ particles. 


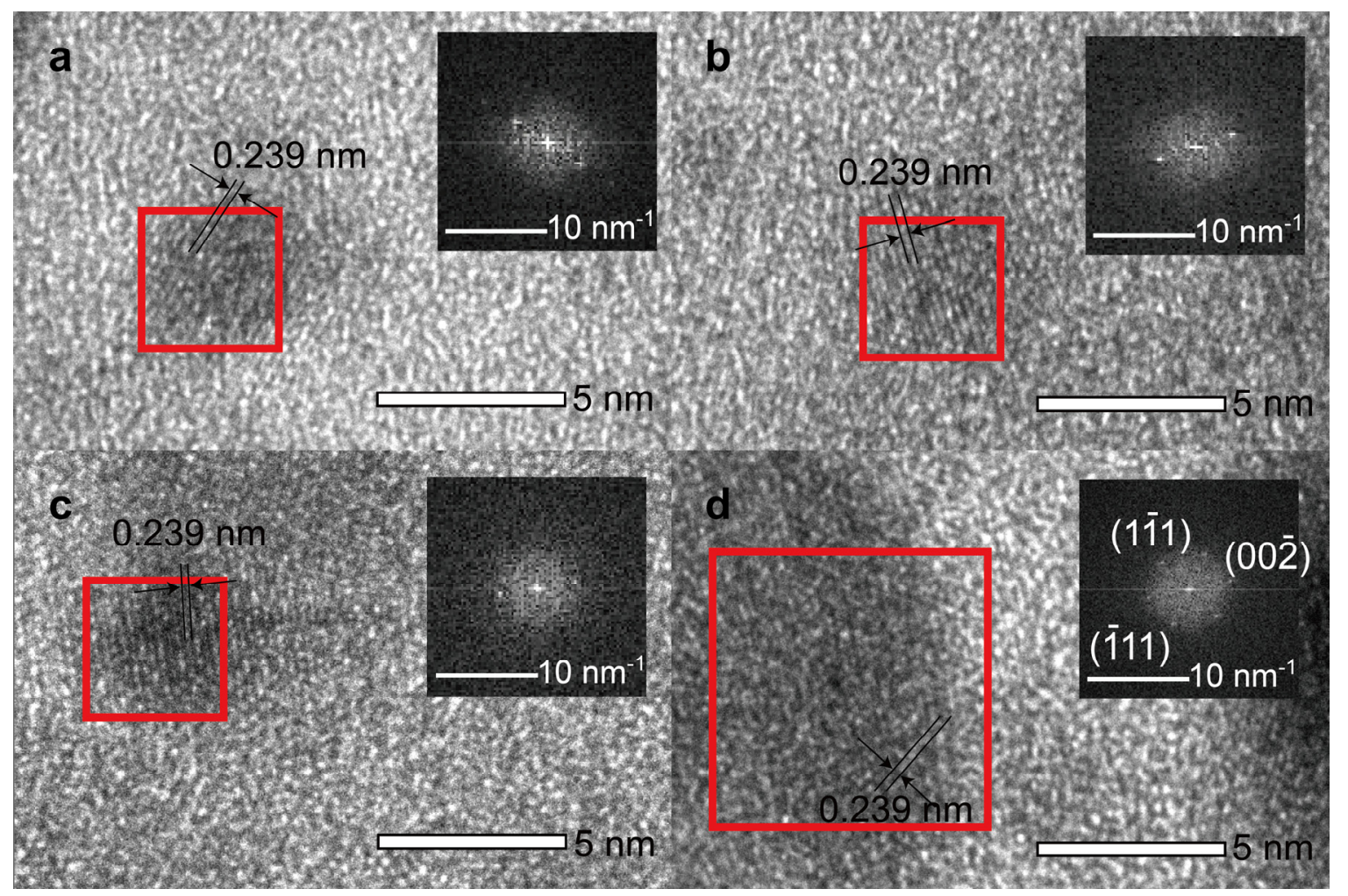

Supplementary Figure S2 HRTEM of Ag nanoclusters in $\mathrm{SiO}_{\mathrm{x}} \mathrm{N}_{\mathrm{y}}: \mathrm{Ag}$ film. The lattice fringes are indexed to $\mathrm{Ag}$ (111) plane in (a) (b) and (c). The (d) indicates face-centered cubic structure of the embedded Ag nanoparticle. 


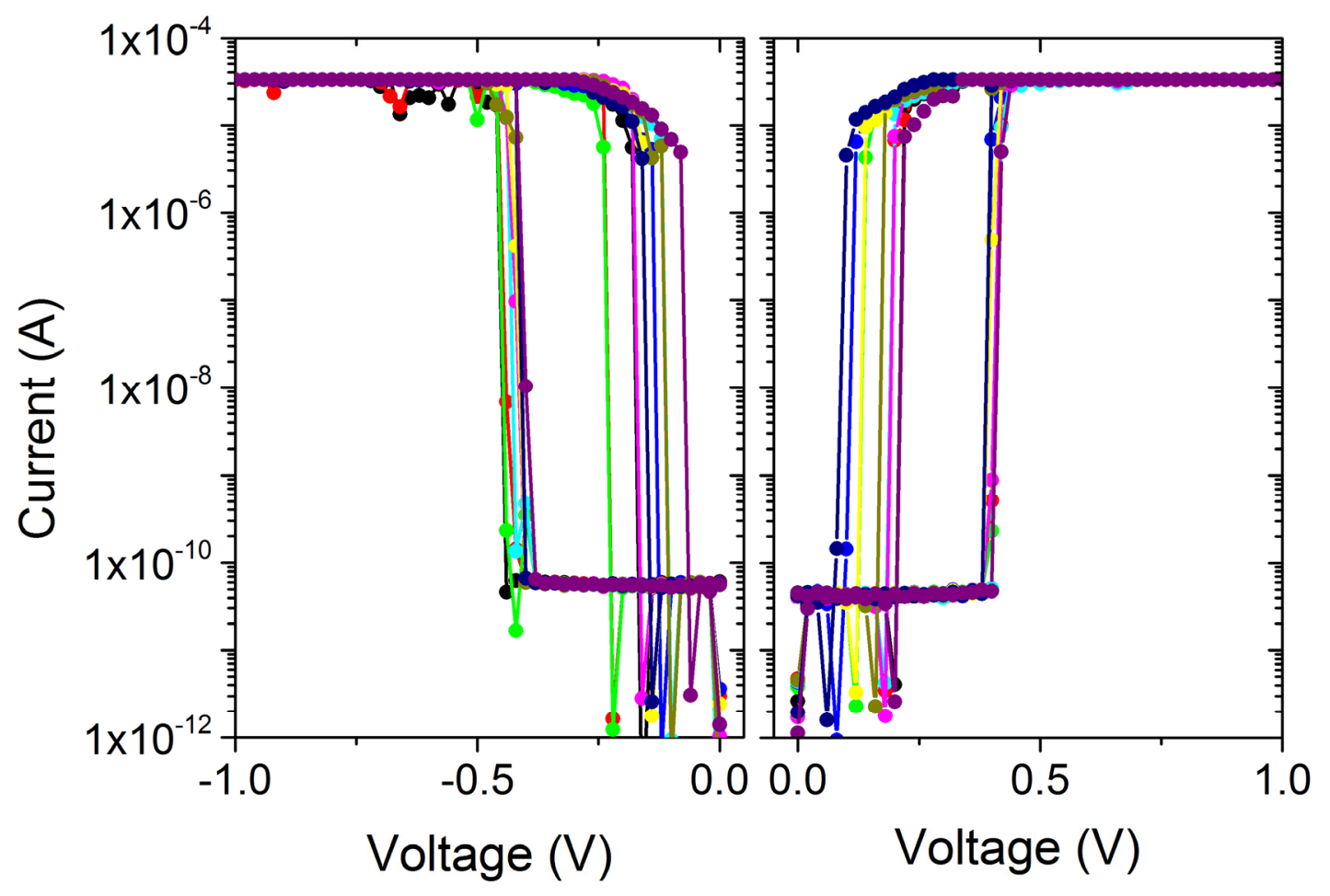

Supplementary Figure S3 The bipolar threshold switching current-voltage characteristics of a crossbar $\mathrm{Pt} / \mathrm{SiO}_{\mathrm{x}} \mathrm{N}_{\mathrm{y}}: \mathrm{Ag} / \mathrm{Pt}$ diffusive memristor device. 


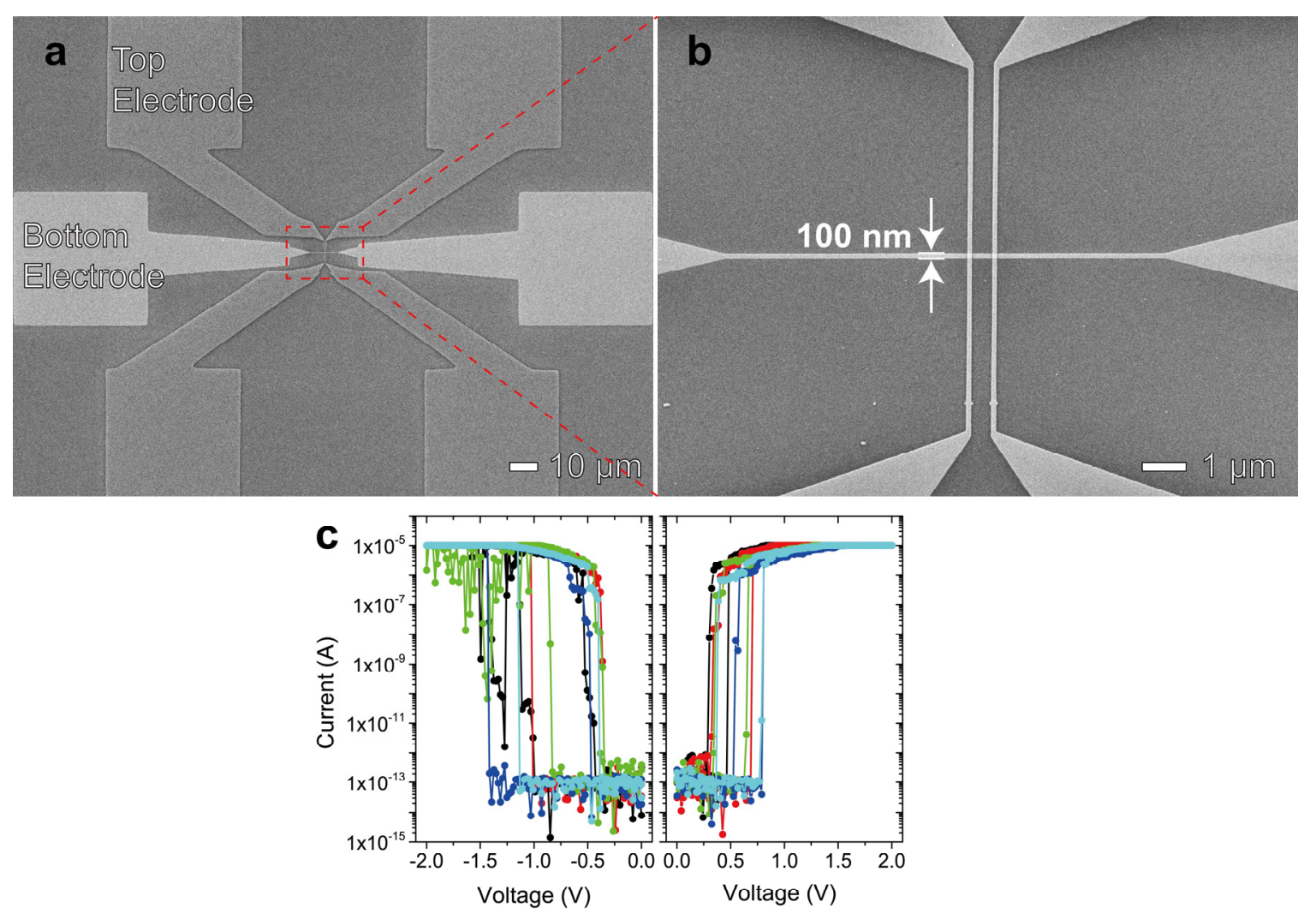

Supplementary Figure S4 Nanoscale crossbar $\mathrm{Pt} / \mathrm{SiO}_{\mathrm{x}} \mathrm{N}_{\mathrm{y}}: \mathrm{Ag} / \mathrm{Pt}$ diffusive memristor and its electrical performance. (a-b) SEM images of the nanoscale crossbar junctions. (c) The currentvoltage characteristics of the device. 

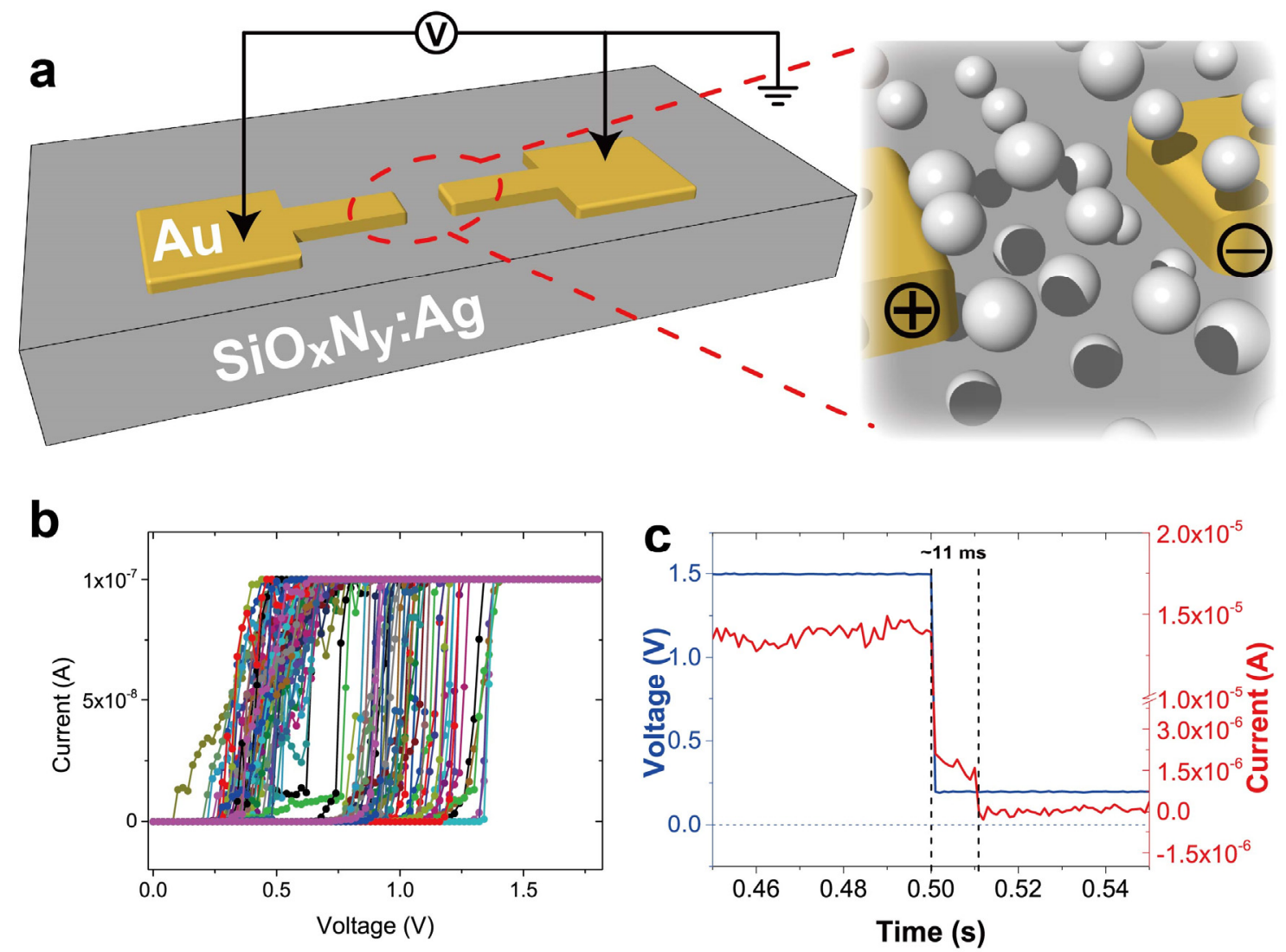

Supplementary Figure S5 Electrical characteristics of $\mathrm{Au} / \mathrm{SiO}_{\mathrm{x}} \mathrm{N}_{\mathrm{y}}: \mathrm{Ag} / \mathrm{Au}$ lateral diffusion memristor (a) Schematic of the planar structure with two gold electrodes embedded in the dielectrics employed for in situ HRTEM. The inset shows the clustered Ag within the gap region serving as bipolar electrodes under biasing. (b) The DC current-voltage characteristics showing repeatable threshold switching in ambient conditions. (c) A typical plot of the timedependent relaxation of device current when a $2 \mathrm{~V}$ excitation pulse followed by a $0.2 \mathrm{~V}$ reading pulse is applied in ambient condition. 

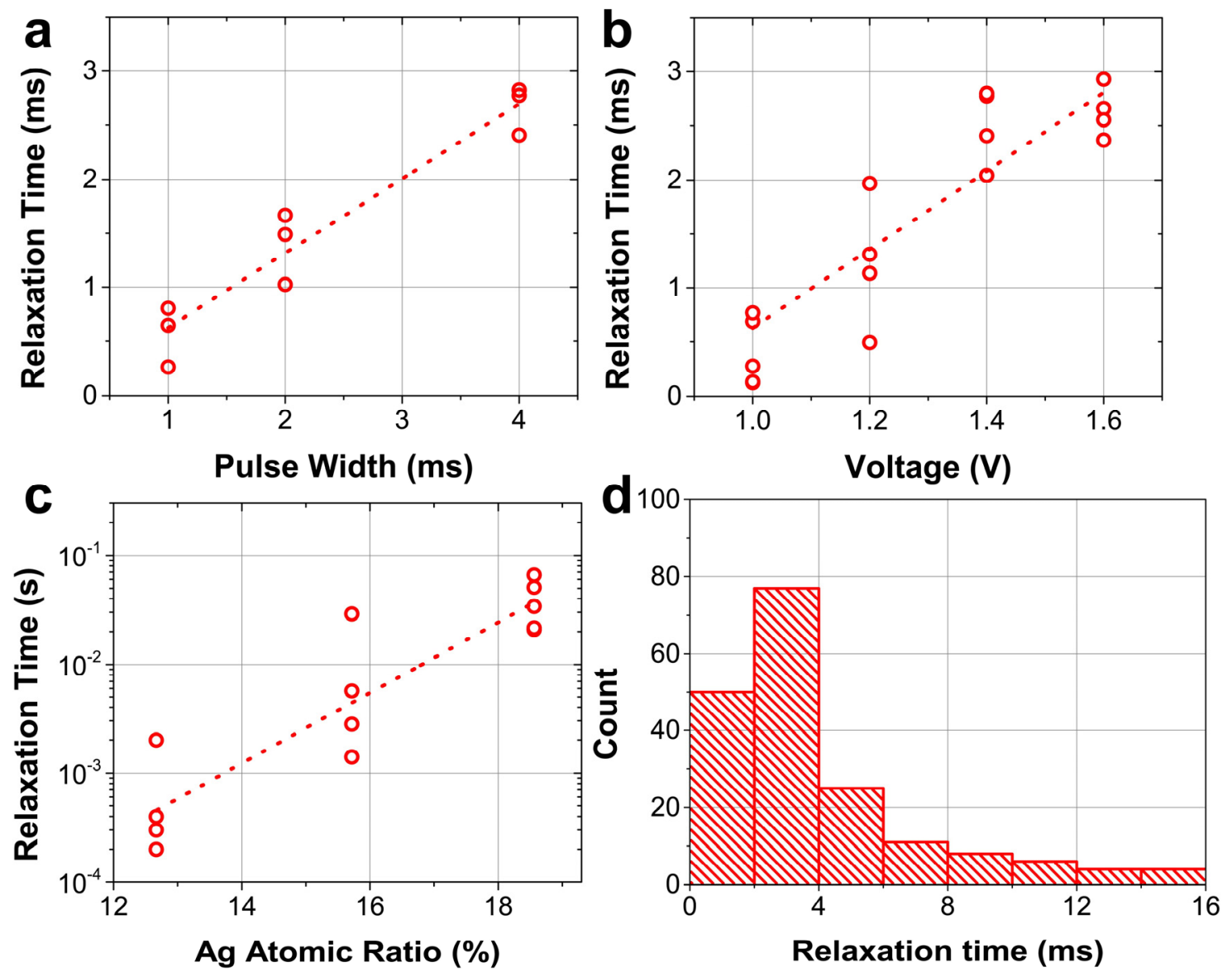

Supplementary Figure S6 The general trend in the variation of relaxation time as a function of pulse width, applied pulse voltage, and the atomic concentration of Ag. (a) The relaxation time increases as a function of pulse width. The pulse voltage used here was $1.4 \mathrm{~V}$. (b) The relaxation time increases with pulse voltage. Here the pulse width was $4 \mathrm{~ms}$. (c) The relaxation time increases with the rising atomic ratio of $\mathrm{Ag}$ in the co-sputtered $\mathrm{SiO}_{\mathrm{x}} \mathrm{N}_{\mathrm{y}}: \mathrm{Ag}$ (see the methods section). The pulse was $1 \mathrm{~V}$ with a $4 \mathrm{~ms}$ width. (d) Histogram plot of the relaxation time of the diffusive memristor extracted from 200 measurements. The applied pulse width and voltage in each independent measurement were $4 \mathrm{~ms}$ and $1.5 \mathrm{~V}$, respectively. 

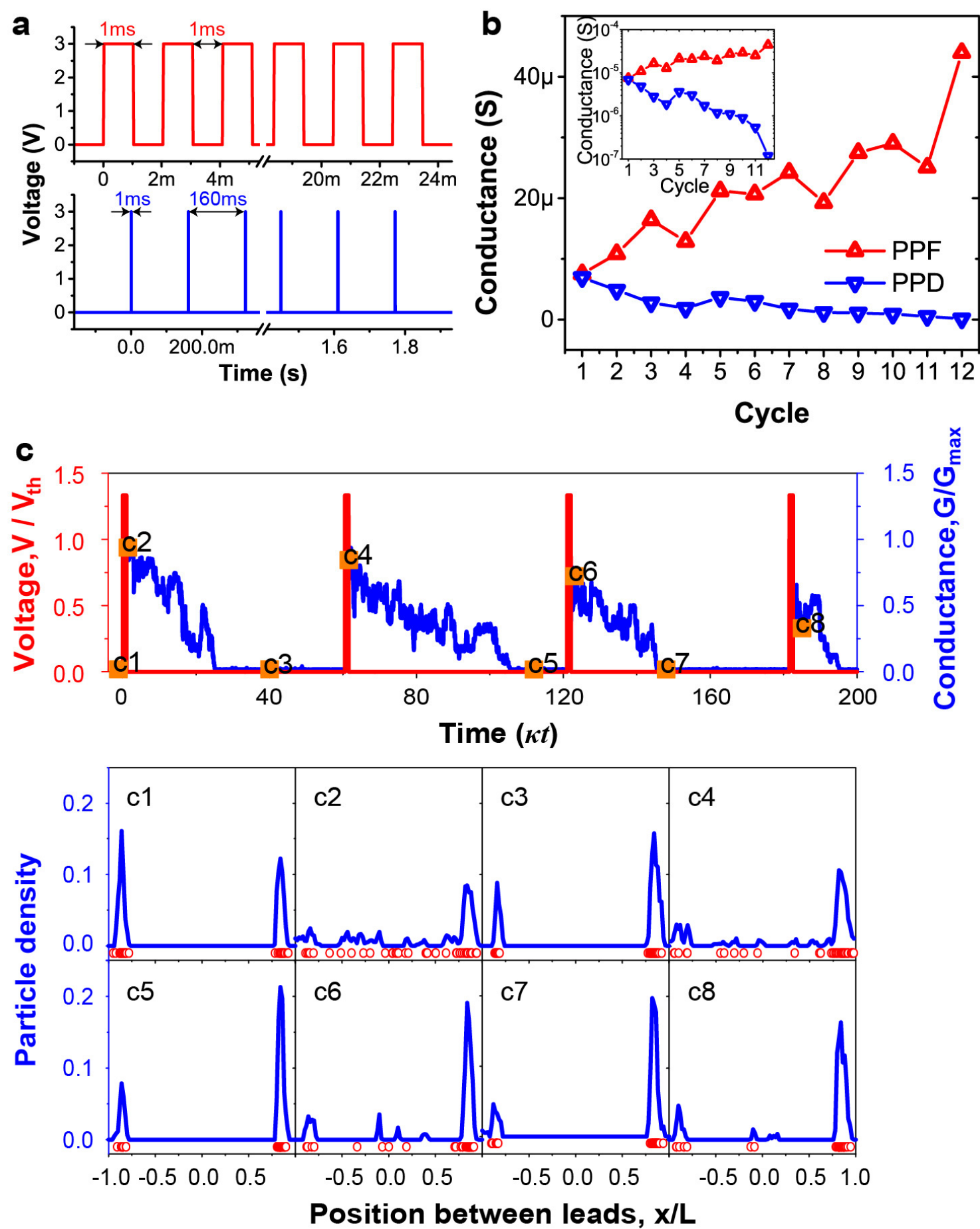

Supplementary Figure S7 Short-term synaptic plasticity with the diffusive $\mathrm{SiO}_{\mathrm{x}} \mathrm{N}_{\mathrm{y}}: \mathrm{Ag}$ memristor (a) Illustration of the voltage pulse train for both PPF and PPD demonstrations. The interval between adjacent $3 \mathrm{~V}$ pulses is $1 \mathrm{~ms}(160 \mathrm{~ms})$ for the PPF (PPD) case. (b) The evolution of conductance as a function of the pulse cycles applied to the device. The device is in its steady state before stimulation in both cases. The conductance increases in the PPF case and decreases in the PPD case. Plotted in the inset is the device conductance in log scale (c) The conductance response (blue curve) of the device due to the higher amplitude and sparse train of pulses (red 
curve) demonstrating PPD; facilitation does not occur since the first pulse excites enough particles to form a bridge between the device terminals and the system completely relaxes to its low conducting state between sequential pulses. Therefore, no excited particle exists between terminals when the next pulse arrives (see, e.g., c1-c3 or c3-c5). The suppression of conductance by the number of pulses still happens due to depletion of left main cluster (compare left and right peaks in the distributions c1, c3, c5, c7), therefore, less and less particles are excited to build a conducting bridge between the terminals (compare c2, c4, c6, c8). Details of simulations and parameters used are described in the methods section and in the caption of Fig. 4 (main text). Note, the depletion saturation can occur due to, e.g., (i) inter-particle repulsion discussed in Ref. 1, and (ii) a diffusion flux against density gradient which is fully taken into account in the model described here. 

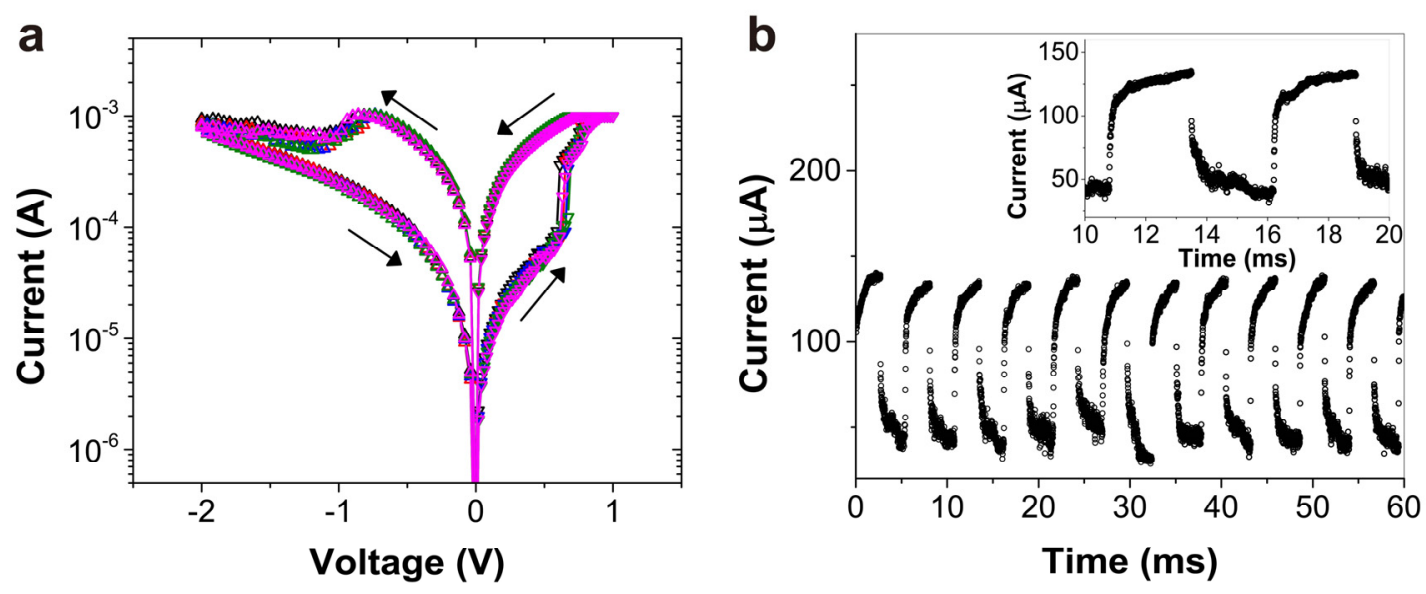

Supplementary Figure S8 Analog switching behaviour of the $\mathrm{Pt} / \mathrm{TaO}_{\mathrm{x}} / \mathrm{Ta} / \mathrm{Pt}$ drift memristors used for the rate-dependent plasticity demonstration of the Figure $6 \mathrm{~b}$ and $6 \mathrm{~d}$ (a) Typical DC current-voltage characteristics of the device. The forming voltage was $\sim 6 \mathrm{~V}$ (b) Pulse based switching response. Plot shows the read current following each pulse in subsequent programming operations on the device. The programming pulse width was $300 \mathrm{~ns}$, the set voltage was $0.7 \mathrm{~V}$, reset voltage was $-1 \mathrm{~V}$, and the read voltage was $0.1 \mathrm{~V}$. 
a
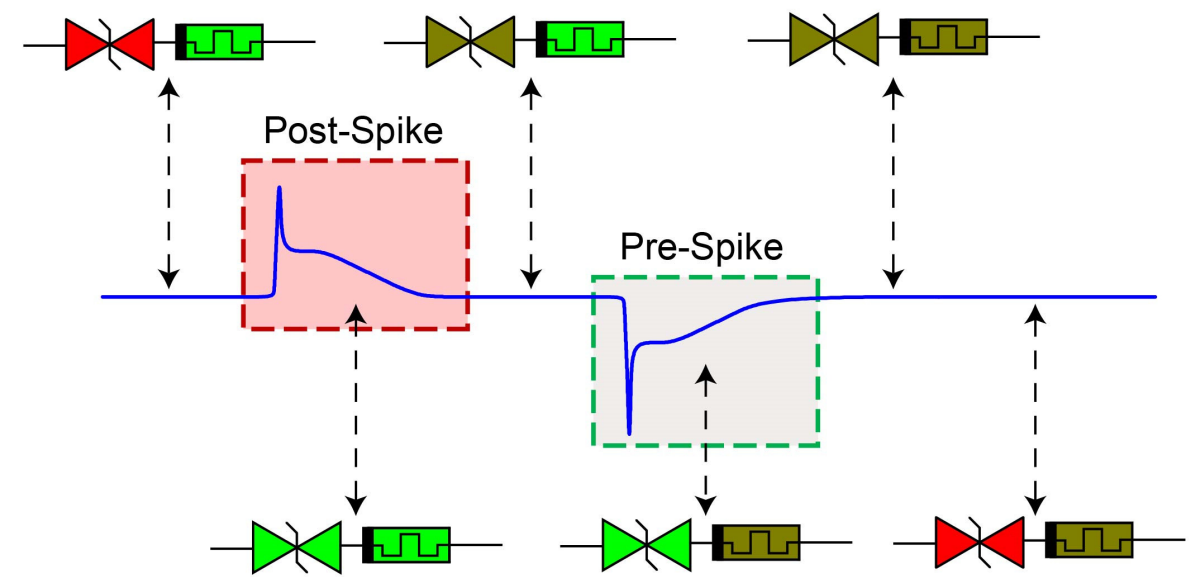

Low Resistance

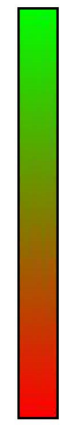

b

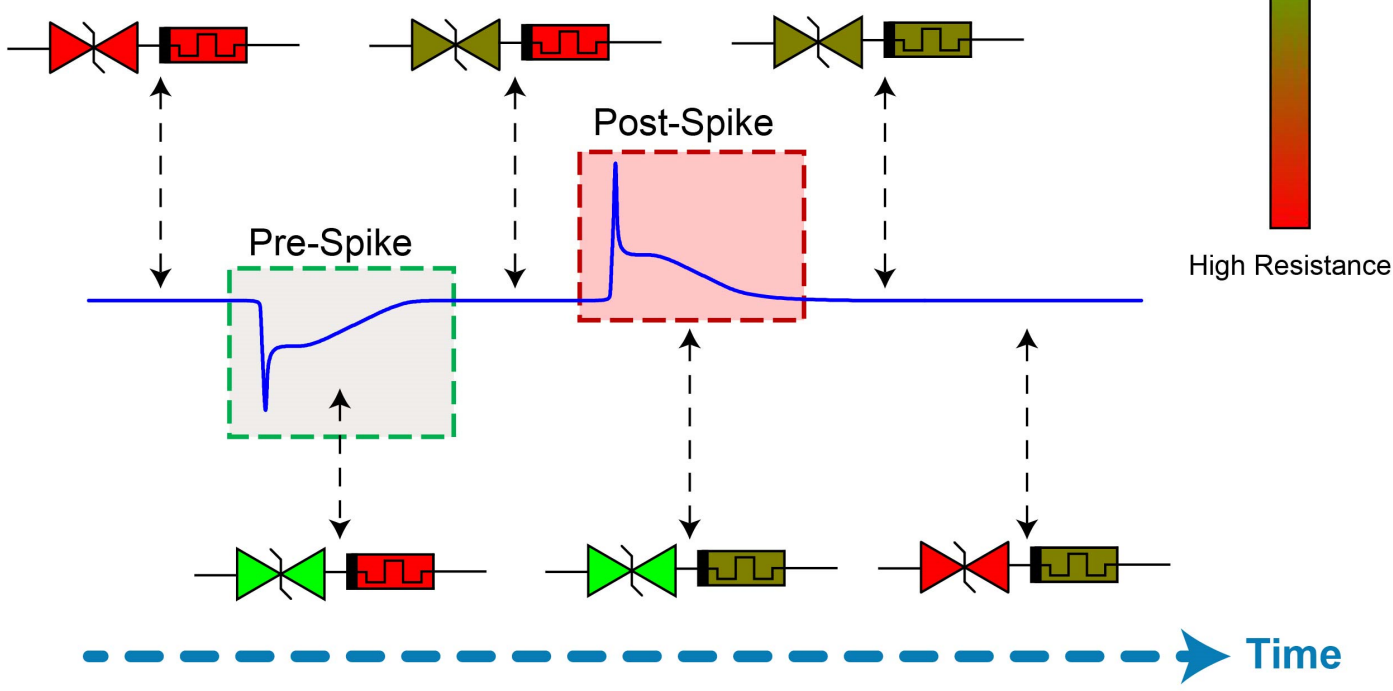

Supplementary Figure S9 Illustration of states evolution in a STDP protocol (a) An example of the evolution of states of both the diffusion and drift memristors during the depression process. The post-spike turns the diffusion memristor ON. The conductance of the diffusion memristor decays to a certain level after the post-spike. The high voltage peak of the pre-spike decreases the conductance of the drift memristor. The conductance of the diffusion memristor decays and it turns OFF after the pre-spike. (b) An example of the evolution of states in the potentiation process. The pre-spike turns the diffusion memristor ON. The conductance of the diffusion memristor decays to a certain level after the pre-spike. The high peak of the postspike increases the conductance of the drift memristor. The diffusion memristor conductance decays and it turns OFF after the post-spike. 

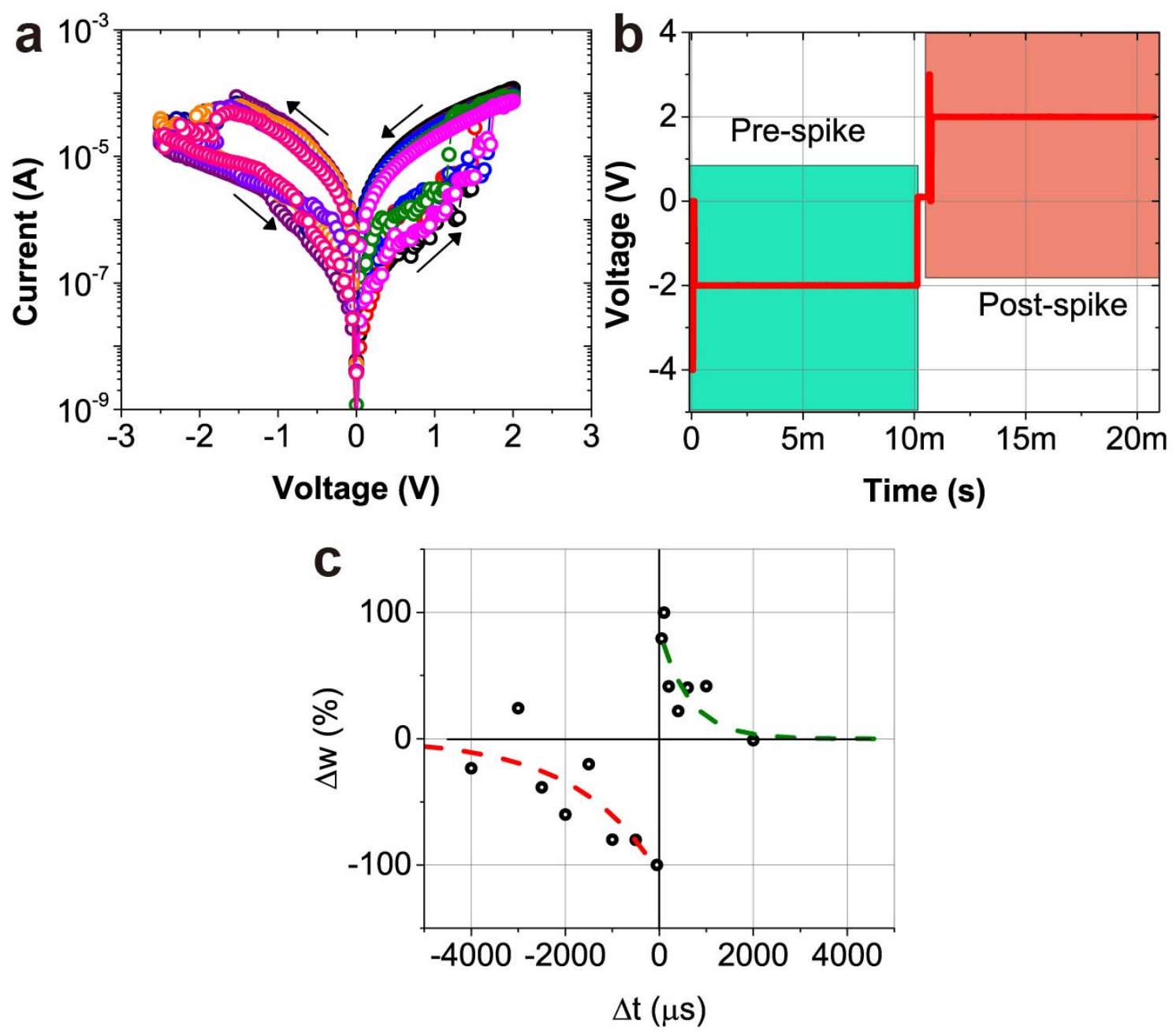

Supplementary Figure S10 STDP with a $\mathrm{Pt} / \mathrm{HfO}_{\mathrm{x}} / \mathrm{TiN}$ drift memristor (a) Typical I-V $\mathrm{Pt} / \mathrm{HfO}_{\mathrm{x}} / \mathrm{TiN}$ drift memristors used for STDP demonstration with a forming voltage of $\sim 4 \mathrm{~V}$. (b) An example of the actual pre- and post-synaptic voltage spikes applied across the device for STDP demonstration. The pre-spike consisted of a $50 \mu \mathrm{s} / 4 \mathrm{~V}$ pulse for programming the drift memristor followed by a $10 \mathrm{~ms} / 2 \mathrm{~V}$ pulse for programming the diffusive memristor. The post-spike had a $50 \mu \mathrm{s} / 3 \mathrm{~V}$ pulse followed by a $10 \mathrm{~ms} / 2 \mathrm{~V}$ pulse. We performed five measurements at each time difference $\Delta t$ and an average was calculated to plot the STDP characteristics. In order to normalize the STDP plot to $100 \%$, the weight change was taken to be equal to the ratio of the change in conductance due to programming and the high conductance value. (c) Plot of the conductance (weight) change of the drift memristor with change in $\Delta t$ showing the spike-timing-dependent plasticity of the electronic synapse. This response is characteristic of the timing-dependent response of biological synapses. 


\section{Reference}

1 Yi, W. et al. Quantized conductance coincides with state instability and excess noise in tantalum oxide memristors. Nat. Commun. 7, 11142, (2016). 